\title{
Carotenoids in Bacteria: Biosynthesis, Extraction, Characterization and Applications
}

\author{
Gerson-Dirceu López, ${ }^{1,2,3}$ Gerardo Álvarez-Rivera, ${ }^{3}$ Chiara Carazzone, ${ }^{1 *}$ Elena Ibáñez, ${ }^{3}$ Chad Leidy, ${ }^{2}$
}

and Alejandro Cifuentes ${ }^{3 *}$

1 Laboratory of Advanced Analytical Techniques in Natural Products (LATNAP), Chemistry Department, Universidad de los Andes, Bogotá D.C., Colombia.; gd.lopez@uniandes.edu.co (G.D.L); c.carazzone@uniandes.edu.co (C. C.).

2 Laboratory of Biophysics, Physics Department, Universidad de los Andes, Bogotá D.C., Colombia; cleidy@uniandes.edu.co (C.L.).

3 Laboratory of Foodomics, Institute of Food Science Research, CIAL, CSIC, Nicolás Cabrera 9, 28049, Madrid, Spain.; gerardo.alvarez@csic.es (G.A.R.); elena.ibanez@csic.es (E.I.); a.cifuentes@csic.es (A.C.).

* Correspondence: c.carazzone@uniandes.edu.co (C.C.); a.cifuentes@csic.es (A.C.)

\begin{abstract}
Natural carotenoids are secondary metabolites that exhibit antioxidant, anti-inflammatory and anti-cancer properties. These types of compounds are in high demand by pharmaceutical, cosmetic, textile and food industries, leading to the search for new natural sources of carotenoids. In recent years, the production of carotenoids from bacteria has become of great interest for industrial applications. In addition to carotenoids with C40-skeletons, some bacteria have the ability to synthesize characteristic carotenoids with C30-skeletons. In this regard, a great variety of methodologies for the extraction and identification of bacterial carotenoids has been reported and this is the first review that condenses much of this information. To understand the diversity of these carotenoids, we present their biosynthetic origin in order to focus on the methodologies employed in their extraction and characterization. Special emphasis has been made on high-performance liquid chromatography-mass spectrometry (HPLC-MS) for the analysis and identification of bacterial carotenoids. We end up this review showing their potential commercial use of bacterial carotenoids. This review is proposed as a guide for the identification of these metabolites, which are frequently reported in new bacteria strains. Keywords: Bacterial carotenoids; Cell disruption; HPLC separation; MS analysis; Food and textile applications
\end{abstract}

\section{Introduction}

Naturally occurring carotenoids are produced by eukaryotic cells such as plants, algae, fungi, and prokaryotic cells like bacteria. In addition, some animals such as flamingos accumulate carotenoids in their plumage from their diet (mollusks and crustaceans). These compounds are characterized by a striking range of colorations from red to yellow, through a spectrum of orange tones. Their characteristic colours are caused by, at least, 6 conjugated double bonds present in their polyene chain. [1,2]. The human eye is able to recognize colours within the range of $360-750 \mathrm{~nm}$ (yellow - blue). The ophthalmic colour discrimination threshold approaches approximately $2 \mathrm{~nm}$, and the Visdetector limit is at best $0.1 \mathrm{~nm}$ [3-5]. These natural pigments are derived from isoprenoids and are classified into two main groups: carotenes (hydrocarbon carotenoids) and oxygenated carotenoids (also known as xanthophylls). Carotenes are made of carbon and hydrogen atoms exclusively (e.g. phytoene, lycopene, $\beta$-carotene). Xanthophyll possess oxygen functional groups, including carotenols (e.g., zeaxanthin), carotenals (e.g., $\beta$-apo8 '-carotenal), carotenones (e.g., cantaxanthin), and carotenoid acids (e.g., 4,4'Diaponeurosporenoic acid) [6-8]. To date, more than 1200 carotenoids and carotenoid precursors from 722 organisms have been reported in the Carotenoid Database (http://carotenoiddb.jp/index.html), where 324 are from bacterial sources, 251 carotenoids being exclusive to these microorganisms [9]. There are variations in the numbers and 
types of carbon units, for example, C30 [10-12], C40 [13], C45 [14], and C50 [15-18]. Chains shorter than C30 occur in some bacteria via an alternate pathway. In general, thirty-seven molecules containing C30 carotenoids have been reported [9]. The double bonds in these polyenes allows for the incorporation of environmentally free radicals, and delocalization of charges (positive or negative) along the chains [19]. The antioxidant activities of carotenoids cause them to be very sensitive to light, heat, oxygen, acidic conditions and/or basic conditions.

These compounds exhibit a broad variety of applications. Carotenoids have been reported to have benefits in treating ocular diseases due to their antioxidant, antiinflammatory or anticancer properties [19-22]. Reviews of carotenoid applications in nutraceutical products have demonstrated the potential of these chemicals, involving carotenes such as phytoene and phytofluene and xanthophylls, such as astaxanthin and lutein [22,23]. Natural carotenoids such as astaxanthin (approved by the European Commission as food dye, E161j) and lycopene (E160d) are used as food colorants. In addition, astaxanthin is used as a dietary supplement, formulated as oils and tablets. Thus, the use carotenoids obtained from microorganisms such as Haematococcus pluvialis (microalgae), Xanthophyllomyces dendrorhous (yeast) and Blakeslea trispora (fungi), is an alternative to synthetic carotenoids in the food industry [24-26].

The main drawbacks of these microorganisms are their long cultivation times, and their dependence on climatic conditions. For example, astaxanthin production by $H$. pluvialis requires up to 288 hours of culture and 156 hours in X. dendrorhous. In addition, microalgae depend on light for the production of carotenoids $[27,28]$. Therefore, research on bacterial carotenoids has become an area of interests due to the short culture times and better yields compared to other microorganisms [29]. Thus, astaxanthin is obtained from the bacteria Halobacillus trueperi MXM-16 and Exiguobacterium Sps after 48 and 72 hours, respectively [30,31]. Although bacteria are typically associated with human pathologies, carotenoids from bacteria are just as safe as those obtained from conventional sources such as plants and chemical synthesis. Thus, the bacterial carotenoids could also be used in pharmaceuticals, textiles, cosmetics, and food [32]. Previous reviews mainly deal with carotenoids produced by yeasts, algae, and fungi over bacteria [33-37], or are maily focused on the applications of these molecules [38-43]. As far as we know, this is the first review about bacterial carotenoids that include chemical aspects such as the biosynthesis, protocols for their extraction, separation and analysis (see Figure 1).

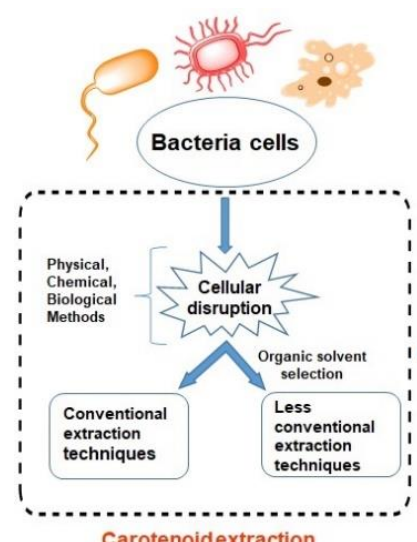

Carotenoidextraction

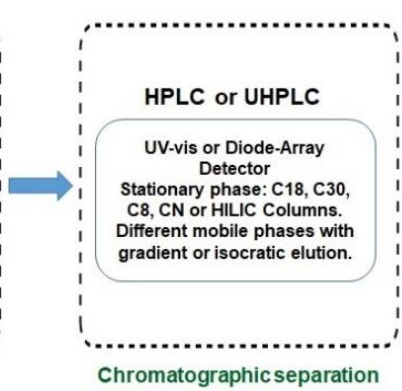

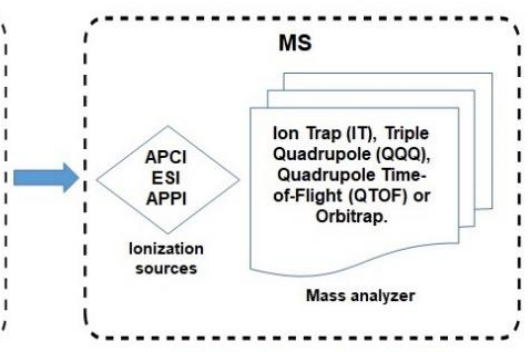

Mass spectrometry analysis

Figure 1. Schematic representation of the steps used for the characterization of bacterial carotenoids.

\section{Biosynthesis of carotenoids}

Carotenoids are secondary metabolites of plants and microorganisms; however, they are not involved in fundamental survival processes such as growth, development, and reproduction. They are associated with the ability to regulate the absorption of light (photooxidation), and other stress response activities [23,44,45]. Besides C40 carotenoids, 
some bacteria can synthesize C30 carotenoids and, to a lesser extent, other C45, C50, C60 chains lengths.

The biosynthesis of the carotenoids derived from bacteria starts from the C5 isoprenoid known as isopentenyl pyrophosphate (IPP), which isomerizes into dimethylallyl pyrophosphate (DMAPP) in the mevalonate (MVA) pathway (Figure 2). Initially, a condensation reaction occurs between one IPP molecule and one DMAPP molecule, producing C10-geranyl diphosphate (GPP). However, depending on the bacteria, additional units of C5 may be added from the 2-C-methyl-D-erythritol-4phosphate (MEP) pathway $[1,6,33]$. Both MAV and MEP are synthesized during bacterial glycolysis. The first species initiating from acetyl CoA, and the second species derived from pyruvate. Chain elongation occurs during successive cyclical reactions, and is catalyzed by prenyl transferase (CrtE). This occurs between one or two IPP molecules with GPP, which produces C15-farnesyl pyrophosphate (FPP) and C20-geranylgeranyl diphosphate (GGPP), respectively. As a result, carotenoids derived from C30 and C40 chains take separate pathways. The synthesis of the first group occurs through the addition of two FPP units, while the second synthesis is due to two GGDP units [38,46,47]. In the C30 carotenoid group, a colourless carotenoid precursor known as 4,4'diaphophytoene is produced, and in the C40 group the 15-cis-phytoene is the first product that is synthesized. This product is also colourless and is catalyzed by the CrtM and CrtB enzymes respectively. In both pathways, successive desaturation processes are carried out by the enzyme desaturase $\mathrm{CrtN}$ (C30) and CrtI (C40). In addition, Figure 2 shows the similarity in the initial steps for the biosynthetic pathways of C30 and C40 carotenoids. While the carotenoids in the C30 group are functionalized early by the oxidation processes, which produce aldehyde, ketone and/or carboxyl groups, carotenoids from the C40 group undergo dehydrogenation and cyclization processes prior to these oxidation reactions [43]. Subsequently, addition, elimination, substitution, or rearrangement reactions will produce a variety of molecules, including stereoisomers (cis/trans) and optical isomers (R/S) [47-50].

Lycopene is the first coloured carotene that is generated from four successive desaturations in the phytoene molecule. Through cyclic reactions, lycopene produces $\alpha$ carotene and $\beta$-carotene, while a hydroxylation reaction in one of the $\beta$-carotene rings produces a $\beta$-cryptoxanthin molecule (Figure 2) [51]. These three pro-vitamin A carotenoids are necessary for retinol synthesis in animals. One molecule of $\beta$-carotene generates two molecules of Vitamin A, a retinoid. This vitamin is necessary for vision, cellular communication, immune function, and human reproduction [20,52,53]. Due to the poor absorption-conversion capacity of vitamin A by human body, the intake of food containing this carotenoid increases its bioavailability in the human digestive system. Xanthophylls, such as lutein, are produced by hydroxylation from $\alpha$-carotene, and they have been identified in the human retina as macular pigments [54]. In addition, it has been proposed that, due to its antioxidant characteristic, lutein may be involved in filtering light that is harmful to the eye $[20,21,52,55]$. 


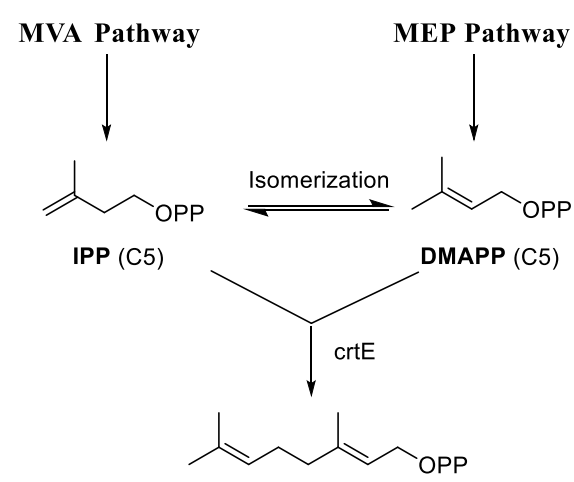

GPP (C10)

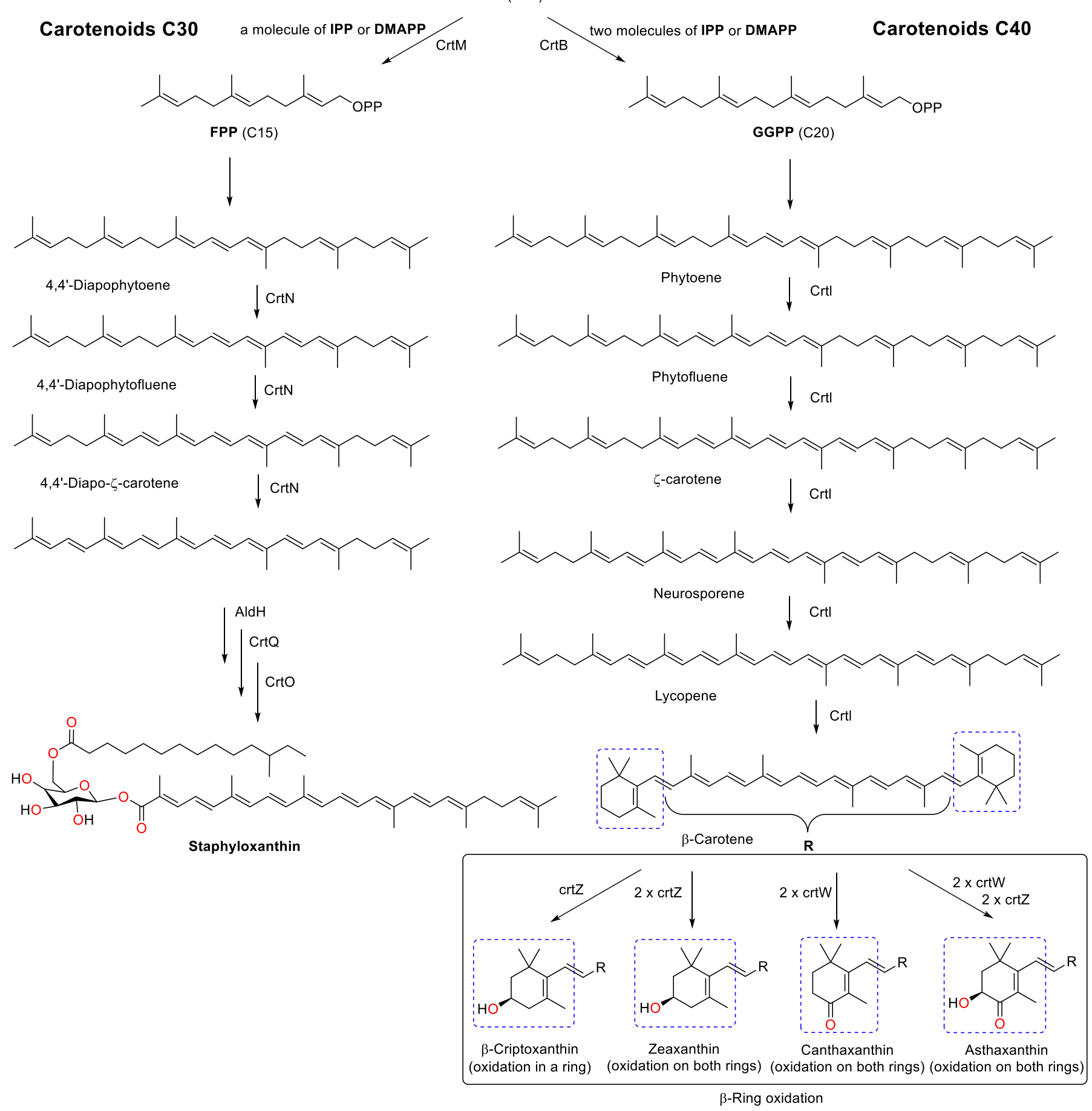

Figure 2. Scheme of bacterial carotenoids biosynthesis with $\mathrm{C} 30$ and $\mathrm{C} 40$ carbon units. 


\section{Extraction Methods}

There is no a single widely accepted method for extracting carotenoids from bacteria, since the yield will depend on different factors (e.g., polarity of carotenoids, moisture content, and kind of cell wall [33,56]). The extraction of carotenes is favoured by the use of non-polar solvents, such as hexane, while xanthophylls are best extracted with polar solvents, such as acetone or ethanol. In addition, the moisture of the bacterial cells will determine whether the extraction is performed by a liquid-liquid or solid-liquid process. The cell wall present in bacteria is another important factor to consider during the extraction procedure. Gram-negative bacteria have inner and outer membranes separated by a thin peptidoglycan layer and are decorated in the outer membrane by lypopolysacharide (LPS); making them generally more permeable to organic solvents. Gram-positive bacteria have an inner membrane covered by a robust cell wall composed primarily of a peptidoglycan network, making these structures more resistant to organic solvents [33]. In both cases, cell disruption of bacteria is simpler compared to microalgae and yeast, which are composed of more complex and rigid cell walls. Figure 3 shows a representation of zeaxanthin in the bacterial membrane of Pantoea sp. YR343 [57]. Thus, cell disruption is required for the extraction of carotenoids from intact bacterial cells (Figure 3, bottom left). Zeaxanthin is also found in lipid extracts used in the preparation of vesicles (Figure 3, bottom right). As a result, the extraction of these metabolites follows a similar process, which starts with cellular disruption or cell-membrane permeabilization, followed by carotenoid solubilization with organic solvent, and further removal of unwanted compounds [2]. In addition, saponification processes are also carried out during the extraction of carotenoids, as they are often bound to sugars, proteins, and other biomolecules such as fatty acids, the latter being very common in bacterial membranes. Extractions can be performed by using conventional or modern techniques.

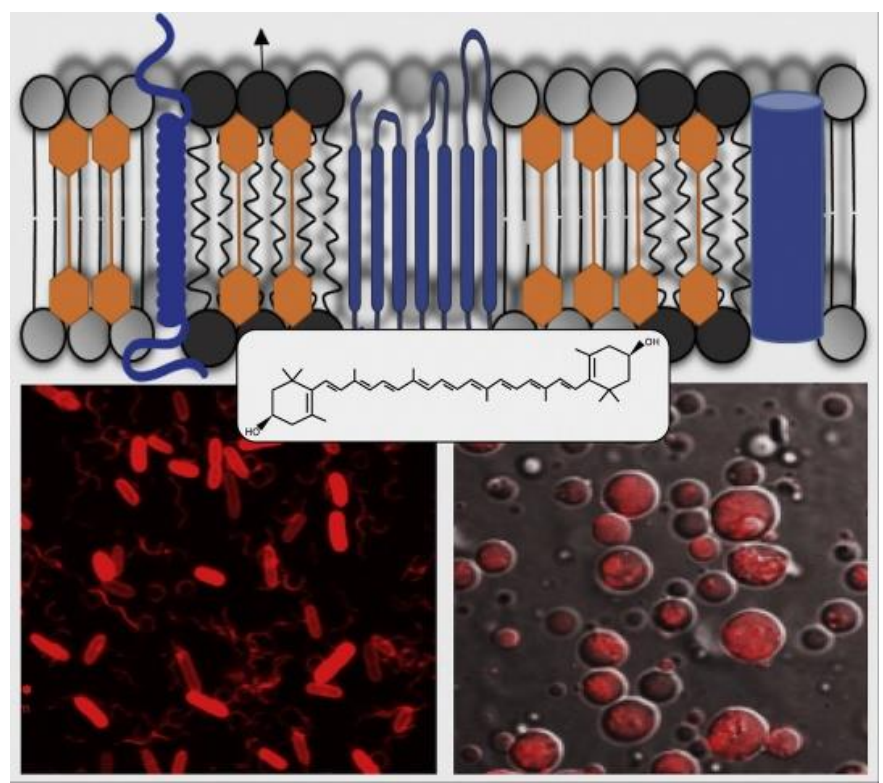

Figure 3. Representation of zeaxanthin in the bacterial membrane of Pantoea sp. YR343 (top of the figure). Intact bacterial cells (lower left) and vesicles prepared from bacterial lipids (lower right), both observed with confocal microscopy. Reprint from Ref. [57].

\subsection{Cellular disruption}

Biomolecules such as proteins, sugars, and fatty acids can lead to multiple problems during the extraction of carotenoids. Thus, cell disruption is a crucial step before the extraction process. However, cell disruption involves an increase in temperature, where thermolabile compounds such as carotenoids can be affected by degradation [58]. After pelleting, cell disruption is carried out by physical, chemical, and/or biological methods 
[16]. Use of mortar and pestle, vortex mixing with or without glass beads, orbital shaking or incubation (may include temperature rise) are examples of physical methods. These approches facilitate the entry of organic solvent into bacteria, leading to solubilization of carotenoids [26,33]. Whereas chemical methods use acids, bases, or surfactants, biological methods use enzymes to cause cell disruption. Extraction techniques include conventional and non-conventional processes that may occur simultaneously during the cellular disruption. Atmospheric liquid extraction with maceration or Soxhlet extraction are typical examples of conventional techniques. Nevertheless, less conventional techniques such as microwaves (microwave-assisted extraction: MAE), ultrasounds (ultrasoundassisted extraction: UAE), supercritical fluid extraction (SFE), pressurized liquid extraction (PLE), and gas expanded liquids (GXLs) may also be employed to simultaneously disrupt and extract carotenoids.

Comparative analysis of three cell disruption methods by means of microwaving, autoclaving, and bead milling in Arthrospira spirulina was conducted [59]. In microwaves, 0.5 grams of cells were used at a frequency of $2450 \mathrm{MHz}$, with $1400 \mathrm{~W}$ of power for 120 seconds. In the autoclaving process, 5 grams of sample were autoclaved for 30 minutes at $121{ }^{\circ} \mathrm{C}$ of temperature and $200 \mathrm{kPa}$ of pressure. In the bead mill process, $150 \mathrm{~g}$ of $\mathrm{A}$. spirulina cells were ground for 2 hours at $60 \mathrm{rpm}$. Water was removed from the autoclaved samples with a vacuum dryer. In this study, microwaving and bead milling resulted in the best yields for cell disruption. In a different study, cell lysis by sonication was evaluated on Formosa sp. KMW bacterium [60]. When an ultrasonic bath was used, yields of $139.67 \pm 7.00 \mu \mathrm{g} / \mathrm{mg}$ biomass was obtained, while with a probe sonicator the yield was $148.9 \pm 19.5 \mu \mathrm{g} / \mathrm{mg}$. However, the vortex extraction method presented the highest yield of carotenoids with $1.83 \pm 0.27$ and $0.96 \pm 0.9 \mathrm{mg} / \mathrm{L}$ respectively. Recently, Park et al. performed cell lysis on freeze-dried cells of the cyanobacterium Arthrospira platensisis [61]. For this extraction method, they used a pre-cooled mortar and acetone containing 0.01\% butylated hydroxytoluene as antioxidant.

Another study evaluated three cell lysis methodologies in Rhodobacter sphaeroides. including: acidic hydrolysis with $\mathrm{HCl}$, grinding, and ultrasounds-assisted extraction [62], with total carotenoids extraction yields of $4650 \mu \mathrm{g} / \mathrm{g}, 1615 \mu \mathrm{g} / \mathrm{g}$ and $645 \mu \mathrm{g} / \mathrm{g}$, respectively. Thus, optimized conditions for $\mathrm{HCl}$-assisted disruption of cell walls $\left(30^{\circ} \mathrm{C}\right.$, solvent-solid ratio of 30:1, for $40 \mathrm{~min}$ ) was the best methodology. In addition, a study on the extraction of carotenoids from cyanobacteria Synechococcus sp. using supercritical $\mathrm{CO}_{2}$ (SFE) was performed [63]. According to this study, the best extraction yields with SFE were achieved by applying 500 bar pressure at a temperature of $60^{\circ} \mathrm{C}$. However, using $15 \%$ ethanol as extraction cosolvent in SFE, resulted in milder conditions to complete the extraction: 300 bar pressure at a temperature of $50^{\circ} \mathrm{C}$. These conditions show a higher relative extraction yield compared to the conventional dimethylformamide (DMF) method. Moreover, high specificity was found in the extraction of carotenoids using SFE compared to DMF, which also extracts high amounts of chlorophylls from cyanobacteria. Comparison of cell disruption by sonication or surfactant treatment did not show a significant increase in carotenoid extraction. Therefore, the most efficient cell disruption method of Synechococcus sp. is achieved through the combination of pressure, temperature and ethanol [63].

Similarly, cell disruption in Rhodothermus marinus was achieved by pressurizing in a PLE cylinder, thus, obtaining hydroxy- and free salinixanthin [64]. Because the bacterium is Gram-negative, the authors of this study did not report a pre-treatment of this bacterium; necessary for the solvent to permeabilize the cells. However, recent results obtained in our lab, using ethyl acetate, demonstrate that macerating the cell-pellet with mortar and pestle is necessary prior to the extraction of Staphyloccocus aureus' (Grampositive bacterium) carotenoids by PLE process. Regarding enzymatic degradation of the cell wall, lysozyme has been used as a biological method in the cell lysis of Formosa sp. KMW and Vitellibacter sp. NMW [65]. Initially, the cells were suspended in a saline solution followed by the addition of the enzyme to proceed with the lysis $(20 \mathrm{mg} / \mathrm{mL})$. Finally, the addition of acetone allowed the solubilization of the carotenoids. Thus, cell disruption is 
a key step that prepares the walls of bacteria for an improved performance in the subsequent or simultaneous extraction process.

\subsection{Organic solvent selection}

During or after cellular disruption, several solvents, such as hexane, acetone, dimethyl sulfoxide (DMSO), dichloromethane (DCM), ethyl acetate (AcOEt), methanol $(\mathrm{MeOH})$ or ethanol $(\mathrm{EtOH})$ can be used for the extraction of bacterial carotenoids, either individually or combined (see Table 1) [2,16]. The selection of the most appropriate organic solvent for each extraction will depend on the type of carotenoids present in the bacteria, as indicated above (xanthophyll or carotenes). Some bacteria only produce carotenes such as $\beta$-carotene, lycopene, etc. Therefore, the choice of the extraction solvent may be easier. However, bacteria that simultaneously produce carotenes and xanthophylls, including their esters, make the solvent selection step more complex. Therefore, a wide variety of solvent combinations has been used to obtain carotenoids from different types of samples [7], making it difficult to propose a standardized solvent or solvent mixture for a given extraction. In addition, the high oxidation sensitivity of carotenoids must be taken into account, bringing about the use of ascorbic acid, butylhydroxytoluene (BHT), butyl-hydroquinone (TBHQ), and butyl-hydroxyanisol (BHA), as antioxidants which stabilize carotenoids during the extraction process $[2,16,66]$. Very often, the analysis of bacterial carotenoids is complex because of their tendency to form ester. Therefore, the hydrolysis of the carotenoid esters before the analysis is frequently required.

\subsection{Saponification}

Bacterial carotenoid extracts contain carotenes, carotenols, carotenoid esters, carotenoid glycosides and carotenoids [7,67]. Carotenoid esters are more stable compared to carotenoids because of the increased hydrophobicity of the molecules, which prevents degradation by oxidation or isomerization due to high temperature conditions and light exposure. Furthermore, with the increase in the degree of esterification of xanthophyll, higher stability of these compounds is observed. For example, lutein is less stable than its monoester, which is less stable than the diester [67]. Despite the stability of xanthophyll esters, their identification is not easy, and a saponification process is required during sample extraction, allowing the analysis of carotenoids. Thus, saponification is an example of a chemical method used for cellular disruption and simultaneous extraction of carotenoids.

Saponification of Bacillus spp. cells was performed with a freeze-dried sample suspended in a solution of $\mathrm{NaOH}(10 \% \mathrm{w} / \mathrm{v})$ that was sonicated for 15 to 20 minutes at room temperature [12]. Subsequently, $\mathrm{NaOH}$ was removed by centrifugation and the carotenoids of the saponified extract were obtained with $\mathrm{MeOH}: \mathrm{CHCl}_{3}$ (1:2) and a Trisbuffer saline. After saponification, the identification of carotenoids was easier than without saponification, showing both lycopenoate and apo-8'-lycopenoate bonded only to glucoside. In the same way, confirmation of an intermediate species in the biosynthetic pathway of $S$. aureus carotenoids was accomplished by performing a saponification of the lyophilized cells with a solution of methanolic $\mathrm{KOH}(6 \%)$ at $4{ }^{\circ} \mathrm{C}$ for 14 hours [68]. This was followed by centrifugation of $4000 \mathrm{rpm}$ at $4^{\circ} \mathrm{C}$ and dried up by centrifugal evaporator. Afterwards, the extract was resuspended in $\mathrm{AcOEt}$ and $\mathrm{NaCl}$ solution $(5.0 \mathrm{~N})$ for removal of unwanted compounds. The by-product 4,4'-Diaponeurosporenoic acid was found both in $S$. aureus and E. coli recombinant bacteria. Since wild-type E. coli does not produce carotenoids, 4,4'-diaponeurosporenoic acid was obtained after insertion of genes from $S$. aureus into $E$. coli bacteria.

A comparative study of different temperatures and $\mathrm{KOH}$ concentrations was carried out in order to evaluate the appropriate combination for carotenoids saponification in microalgae [69]. Thus, the solvent mixture EtOH:hexane:water (77:17:6) with 0-60\% $\mathrm{KOH}$ $((\mathrm{g} \mathrm{KOH} / \mathrm{g}$ dry biomass $) \times 100)$ at a temperatures between 25 and $80{ }^{\circ} \mathrm{C}$ were tested. 
Although the results did not allow to establish a widely applicable method for the extraction of carotenoids from the eight microalgae studied, ideal conditions were found for each strain. The researchers found that $P$. reticulatum requires a temperature of $40{ }^{\circ} \mathrm{C}$ and $10 \% \mathrm{KOH}$, while the best yields for T. suecica and $\mathrm{H}$. pluvialis are achieved at $25^{\circ} \mathrm{C}$ and $10 \%$ or $40 \% \mathrm{KOH}$, respectively. In I. galbana the ideal temperature was $60{ }^{\circ} \mathrm{C}$ and $<10 \%$ $\mathrm{KOH}$, while Chlorella sp. and S. almeriensis require a temperature of $80{ }^{\circ} \mathrm{C}$ and $40 \% \mathrm{KOH}$. Finally, for $N$. gaditana and $K$. veneficum, the best results were obtained without saponification at $60{ }^{\circ} \mathrm{C}$. Therefore, it is not possible to establish standardized saponification conditions, since these conditions depend on the target strain, which can be extended to other microorganisms such as yeasts and bacteria.

\subsection{Conventional extration techniques}

Conventional techniques for carotenoids extraction have been reported in different studies. For example, S. aureus was incubated at 40 or $4{ }^{\circ} \mathrm{C}$ for 20 to 30 minutes, using $\mathrm{EtOH}, \mathrm{MeOH}$ or acetone was used in the liquid-liquid extraction process [68,70]. Also, a secondary liquid-liquid extraction with a AcOEt: $\mathrm{NaCl}$ aqueous solution allow the cleaning of the extract from the very polar compounds by a salting-out effect. In both cases, several related intermediaries of the staphyloxanthin (STX) biosynthetic pathway or new carotenoid species in mutant strains were determined [68]. STX is a saccharolipid derivate from a 4,4'-diaponeurosporenoic acid, which is associated with increased membrane stiffness and resistance to antimicrobial peptide activity [71]. These studies have helped decipher the carotenoid biosynthesis in this human pathogen, allowing for the identification of a potential target for anti-virulence therapy [10]. Similarly, Rezaeeyan et al. [72] indicated that the extraction of neurosporene (a carotene with antioxidant activity and UV-B radiation protection) from Kocuria sp. QWT-12 can be achieved by incubating with $\mathrm{MeOH}$ at $60^{\circ}$ for 15 minutes.

Recently, Hartz el al. [73] studied the carotenoids 4,4'-diapophytoene, 4,4'diapophytofluene, 4,4'-diaponeurosporene, 4,4'-diapolycopene, 4,4'-diaponeurosporenic acid from Bacillus megaterium using a cold extraction method with $\mathrm{MeOH}\left(-20^{\circ} \mathrm{C}\right)$ combined with vortex and glass beads. This method prevents thermal degradation of carotenoids, which can occur in the incubation processes. In addition, 4,4'diaponeurosporene is a carotene which has the potential to treat inflammatory diseases. This carotene was selectively extracted from E. coli [74]. A similar disruption of the cells using EtOH was carried out in Methylomonas sp. 16a [75]. This disruption allowed the analysis of astaxanthin and canthaxanthin, that are not naturally produced by this bacterium, but obtained after genetic engineering. These carotenoids have been reported to have photoprotective, antioxidant and anti-inflammatory effects. Also, isolation and characterization of astaxanthin, adonixanthin, hydroxy-astaxanthin and dihydroxyastaxanthin from marine Gram-negative bacteria Brevundimonas sp. [25] and Erythrobacter [13] was possible by extraction followed by shaking at $50{ }^{\circ} \mathrm{C}$. Astaxanthin is characterized by its high antioxidant potential, which is greater than $\beta$-carotene (10 times) and vitamin E (500 times). This health benefit is often used in the field of dermatology [22]. Adonixanthin has been reported to be even stronger than astaxanthin in protecting the brain from internal haemorrhages [76].

Different expeditions have found numerous bacterial strains in Antarctica. A comprehensive study on the carotenoids produced from 30 pigmented bacterial strains isolated from Fildes Peninsula, King George Island, was reported by Vila et al. [77]. These results suggested that the antioxidant activity that carotenoids provide to these bacteria increased the resistance to extreme temperature conditions, drastic light conditions, and high doses of UV-B. Carotenoids such as zeaxanthin, $\beta$-cryptoxanthin and $\beta$-carotene were the main components obtained during extraction with $\mathrm{MeOH}$. The toxicity of the selected solvent must always be considered, in particular, when scaling up the process to the industrial level. Therefore, green extraction techniques arise, which involve the use of more environmentally friendly solvents such as ethanol, ethyl acetate, or water. Due to 
the physicochemical characteristics of carotenoids, water is not a viable option in the extraction of these pigments.

\subsection{Less conventional extraction techniques}

An important aspect that may hinder the industrial application of carotenoids is the need for organic solvents during the extraction procedures, which have adverse effects on the environment and human health. Hence, the research on green chemistry approaches, which usually implies the use of less conventional extraction techniques, is of paramount importance. In addition, these techniques may offer higher yields of carotenoids in shorter periods of time when compared to traditional techniques [56]. For example, when PLE is used for carotenoids extraction, the high yield obtained is due to the higher selectivity of PLE compared to other conventional extraction techniques. As far as we know, only a few examples have been reported using PLE for the extraction of carotenoids from bacteria. Ron et al. employed PLE in the extraction of carotenoids from $R$. marinus strains DSM 4252T, DSM 4253, and PRI 493 [64], using ethanol with 1600 psi of pressure at $100{ }^{\circ} \mathrm{C}$, during 3 extraction cycles of 2 minutes each. Four main xanthophylls were obtained: two carotenoid acyl glycoside (-hydroxyl and free) and salinixanthin (-hydroxyl and free).

Among these methods, UAE seems to be the least effective approach, even though it is the most used technique for the extraction of carotenoids from bacteria. This could be due to the low extraction temperatures used to avoid their degradation. Astaxanthin has been extracted with UAE from Halobacillus trueperi MXM-16 bacterium using a $\mathrm{MeOH}$ :acetone (1:1) mixture[30]. Recently, Buddhi et al. [78] reported the spirilloxanthin series analysis of Afifella aestuarii sp. nov. using UAE with an acetone:MeOH (9:0.5) mixture. Also, Giuffrida et al. [18] using UAE with $\mathrm{MeOH}$ analyzed C50 carotenoids, such as all-E-decaprenoxanthin, all-E-sarcinaxanthin, 9-Z-decaprenoxanthin, 15-Zdecaprenoxanthin produced by strains of the cheese-ripening bacterium Arthrobacter arilaitensis. Extraction of zeaxanthin from Formosa sp. KMW and Vitellibacter sp. NMW was achieved by using a biological method of cell disruption which uses lysozyme with acetone in enzyme assisted extraction [65]. Similarly, cis- $\beta$-carotene, all-trans- $\beta$-carotene, chlorobactene, isorenieratene were obtained from Rhodococcus sp. B7740 employing the same enzyme [79].

Surprisingly, despite the claimed advantages of the MAE, no reports on the use of this technique for carotenoid extraction from bacteria were found in the literature; most probably due to the time-consuming process of optimization, including solvent-sample ratio, solid-liquid ratio, and microwave power [16].

In summary, the wide variety of carotenoids produced by bacteria requires continuous development and optimization of new extraction methods. Once the carotenoids fraction is obtained, chemical characterization of this fraction is usually carried out (see Figure 1) employing HPLC-MS as described below. Table 1 presents detailed information on the methods used for the extraction of bacterial carotenoids, as well as the conditions used in their identification by HPLC-MS. 
Table 1: Carotenoids obtained from different bacteria, extraction conditions and analytical methodologies applied.

\begin{tabular}{|c|c|c|c|c|c|c|}
\hline Carotenoids & Bacteria & Filum & Gram & Extraction Technique & Characterization conditions & Ref. \\
\hline $\begin{array}{l}\text { All carotenoids biosynthetic pathway } \\
\text { Staphyloxanthn. }\end{array}$ & \multirow[t]{3}{*}{ Staphylococcus aureus } & \multirow[t]{3}{*}{ Firmicutes } & \multirow[t]{3}{*}{$\operatorname{Pos}(+)$} & $\begin{array}{l}\text { Cell without lyophilizate }+\mathrm{EtOH}+ \\
\text { incubated water bath } \times 20 \text { min at } 40{ }^{\circ} \mathrm{C}+ \\
\text { centrifugation }+ \text { AcOEt: } \mathrm{NaCl} \text { solution } \\
(3: 1)+\mathrm{Na}_{2} \mathrm{SO}_{4} \text { (Anh.). }\end{array}$ & $\begin{array}{l}\text { HPLC-UV-APCI-MS(IT): } \quad \text { C30 } \\
\text { column } 3 \mu \mathrm{m}(4.6 \times 250 \mathrm{~mm}) \text {; mobile } \\
\text { phase: (A) water, (B) acetone. } \\
\text { Gradient elution. }\end{array}$ & {$[70]$} \\
\hline $\begin{array}{l}\text { Hydroxy-diaponeurosporenal, } \\
\text { diaponeurosporenal, diapolycopenedial, } \\
\text { diapolycopen-al-oic acid. }\end{array}$ & & & & $\begin{array}{l}\text { Cell without lyophilizate }+ \text { acetone }+ \\
\text { incubate ultrasound } \times 30 \mathrm{~min} \text { at } 4{ }^{\circ} \mathrm{C}+ \\
\text { centrifugation }+\mathrm{AcOEt}: \mathrm{NaCl} \text { solution } \\
(5.0 \mathrm{M}) .\end{array}$ & $\begin{array}{l}\text { HPLC-DAD-APCI-MS(IT): } \quad \text { C18 } \\
\text { column } 2.5 \mu \mathrm{m}(4.6 \text { x } 150 \mathrm{~mm}) ; \\
\text { mobile phase: (A) dwater, (B) ACN. } \\
\text { Gradient elution. }\end{array}$ & {$[80]$} \\
\hline $\begin{array}{l}\text { All carotenoids biosynthetic pathway } \\
\text { Staphyloxanthn. }\end{array}$ & & & & $\begin{array}{l}\text { Cell without lyophilizate }+ \text { acetone or } \\
\mathrm{MeOH}+\text { centrifugation }+ \text { centrifugal } \\
\text { evaporator }+ \text { resuspending in AcOEt }+ \\
\mathrm{NaCl} \text { solution }\left(\begin{array}{lll}5.0 & \mathrm{~N})+ \text { dried in } \\
\text { centrifugal } & \text { evaporator. } \\
\text { Saponification: sample }+ \text { incubating in } \\
\text { MeOH at } 6 \% \mathrm{KOH} \times 14 \text { hat } 4{ }^{\circ} \mathrm{C}+\text { similar } \\
\text { steps previously described. }\end{array}\right.\end{array}$ & $\begin{array}{l}\text { HPLC-UV-vis-APCI-MS(IT): } \quad \text { C18 } \\
\text { column } 5 \mu \mathrm{m}(4.6 \times 150 \mathrm{~mm}) ; \text { mobile } \\
\text { phase: ACN:MeOH:IPA (80:15:5). } \\
\text { Isocratic elution. }\end{array}$ & {$[68]$} \\
\hline Apocarotenoides family. & Bacillus sp. & Firmicutes & Pos $(+)$ & $\begin{array}{l}\text { Lyophilizate cell }+ \text { mortar-pestle }+ \\
\mathrm{MeOH}+\text { sonicated at r.t. x } 10-15 \mathrm{~min}+ \\
\text { centrifugation. Repeated extraction until } \\
\text { lack pigment }+ \text { dried in centrifugal } \\
\text { evaporator. Saponification: sample + } \\
\mathrm{NaOH} \text { solution }(10 \%)+\text { sonication } \times 15- \\
20 \text { min at r. t. + centrifugation + } \\
\mathrm{MeOH}: \mathrm{CHCl}_{3}(1: 2)+\text { tris-buffered saline }\end{array}$ & $\begin{array}{l}\text { HPLC-DAD: C30 column } 5 \mu \mathrm{m}(4.6 \\
\text { x } 250 \mathrm{~mm}) \text { with a C30 guard column } \\
(4.6 \times 20 \mathrm{~mm}) \text {; mobile phase: (A) } \\
\text { MeOH, (B) MeOH:water (8:2) with } \\
0.2 \%(\mathrm{w} / \mathrm{v}) \text { ammonium acetate (C) } \\
\text { MTBE. Gradient elution. HPLC- } \\
\text { APCI-MS: C30 column } 3 \mu \mathrm{mm} \\
(2.1 \times 150 \mathrm{~mm}) \text { with a C30 guard } \\
\text { column }(4.6 \times 20 \mathrm{~mm}) ; \text { mobile phase: }\end{array}$ & [12] \\
\hline
\end{tabular}




\begin{tabular}{|c|c|c|c|c|c|c|}
\hline & & & & $\begin{array}{l}\text { + centrifugation + collected carotenoids } \\
\text { in chloroform phase + dried with } \mathrm{N}_{2} \text { gas. }\end{array}$ & $\mid \begin{array}{llll}\text { (A) } & \mathrm{MeOH}, \quad \text { (B) } & \text { MTBE, } & \text { both } \\
\text { containing } & 0.1 \% & \text { formic } & \text { acid. } \\
\text { Gradient elution. } & & \end{array}$ & \\
\hline $\begin{array}{l}\text { 4,4'-diapophytoene, } \\
\text { 4,4'-diapophytofluene, } \\
\text { 4,4'-diaponeurosporene, } \\
\text { 4,4'-diapolycopene, } \\
\text { 4,4'-diaponeurosporenic acid. }\end{array}$ & Bacillus megaterium & Firmicutes & $\operatorname{Pos}(+)$ & $\begin{array}{l}\text { Cell without liofilizate }+80 \% \mathrm{MeOH}(- \\
\left.20^{\circ} \mathrm{C}\right)+ \text { glass beads }+ \text { vortex } 30 \mathrm{~s} \times 5 \\
\text { times }+30 \text { s cool on ice. }\end{array}$ & $\begin{array}{l}\text { HPLC-DAD-ESI-MS (QTOF): HILIC } \\
\text { column } 3.5 \mu \mathrm{m}(2.1 \times 100 \mathrm{~mm}) ; \\
\text { mobile phase: }(\mathrm{A}) \mathrm{ACN},(\mathrm{B}) \text { water } \\
\text { both with } 10 \mathrm{mM} \text { ammonium } \\
\text { acetate }(\mathrm{pH}=6) \text {. Gradient elution. }\end{array}$ & [73] \\
\hline $\begin{array}{l}\text { Glycosyl-4,4'-diaponeurosporen-4'-ol-4- } \\
\text { oic acid, 4,4'-diaponeurosporen-4'-ol-4 oic } \\
\text { acid. }\end{array}$ & Planococcus faecalis AJ003T & Firmicutes & Pos (+) & $\begin{array}{l}\text { Cell without lyophilizate + acetone or } \\
\mathrm{MeOH}+\text { centrifugation }+ \text { centrifugal } \\
\text { evaporator }+\mathrm{AcOEt}: \mathrm{NaCl} \text { solution }(5 \mathrm{~N}) \\
+\mathrm{Na}_{2} \mathrm{SO}_{4} \text { (Anh.) + dried centrifugal } \\
\text { evaporator. }\end{array}$ & $\begin{array}{l}\text { HPLC-DAD: C18 column } 2.5 \mu \mathrm{m} \\
(4.6 \text { x } 150 \mathrm{~mm}) \text {; mobile phase: } \\
\text { (A)dwater, (B) ACN. Gradient } \\
\text { elution. HPLC-DAD-APCI-MS: C18 } \\
\text { column } 5 \mu \mathrm{m}(4.6 \times 150 \mathrm{~mm}) \text {; mobile } \\
\text { phase: (A) ACN:MeOH:IPA } \\
\text { (80:15:5), (B) MeOH:water (7:3) (C) } \\
\text { MeOH. Gradient elution. }\end{array}$ & {$[11,68,81,82]$} \\
\hline Salinixanthin, carotenoid acyl glycoside & Rhodothermus marinus & $\begin{array}{l}\text { Bacteroidetes/chlorobi } \\
\text { group }\end{array}$ & $\operatorname{Neg}(-)$ & $\begin{array}{l}\text { Cells washed and lyophilizate }+\mathrm{EtOH} \text { at } \\
100{ }^{\circ} \mathrm{C} \text { with } 1500 \text { psi } \times 2 \text { min in PLE } \\
\text { (three times) + concentration. }\end{array}$ & $\begin{array}{l}\text { UHPSFC-DAD-ESI-MS: (QTOF), } \\
\text { CSH fluoro-phenyl } 1.8 \mu \mathrm{m}(3 \times 100 \\
\mathrm{mm}) \text {; mobile phase: (A) } \mathrm{CO}_{2} \text { (B) } \\
\mathrm{MeOH} \text { with ammonium formate } \\
(0.2 \%) \text {. Gradient elution. }\end{array}$ & [64] \\
\hline
\end{tabular}




\begin{tabular}{|c|c|c|c|c|c|c|}
\hline $\begin{array}{|lr|}\text { cis-synechoxanthin, } & \text { all-trans- } \\
\text { synechoxanthin, cis- } \beta \text {-carotene, all-trans- } \\
\beta \text {-carotene, chlorobactene, Isorenieratene. }\end{array}$ & Rhodococcus sp. B7740 & Actinobacteria & Pos (+) & $\begin{array}{l}\text { Lyophilized cell + lysozyme }+ \text { vortex } x \\
30 \mathrm{~s}+\text { incubation at } 37^{\circ} \mathrm{C} \times 1 \mathrm{~h}+ \\
\text { centrifugation at } 7000 \mathrm{~g} \text { at } 4^{\circ} \mathrm{C} \times 10 \mathrm{~min} \\
+ \text { extraction with MeOH:DCM (8:2) + } \\
\text { centrifugation. }\end{array}$ & $\begin{array}{l}\text { HPLC-DAD-APCI-MS(D-Trap- } \\
\text { XCT) and HPLC-DAD-ESI- } \\
\text { HRMS(LTQ-Qrbitrap): C30 column } \\
5 \mu \mathrm{m}(4.6 \times 150 \mathrm{~mm}) \text {; mobile phase: } \\
\text { (A) } \mathrm{MeOH} \text {, (B) MTBE. Gradient } \\
\text { elution. } \beta \text {-carotene, lutein and } \\
\text { isorenieraten standards. }\end{array}$ & [79] \\
\hline 4-keto $\gamma$-carotene, $\gamma$-carotene. & $\begin{array}{l}\text { Rhodococcus erythropolis } \\
\text { AN12 }\end{array}$ & Actinobacteria & Pos (+) & Cell pellets + acetone & $\begin{array}{l}\text { HPLC-DAD: C8 column }(4 \times 125 \\
\text { mm); mobile phase: (A) MeOH (B) } \\
\text { water. Gradient elution. HPLC- } \\
\text { APCI-MS(QqQ): C18 column } \\
(4.6 \times 250 \mathrm{~mm}) \text {; mobile phase: ACN: } \\
\text { acetone (3:1). Isocratic elution. } \beta- \\
\text { carotene, } \gamma \text {-carotene and lycopene } \\
\text { standards. }\end{array}$ & {$[83,84]$} \\
\hline $\begin{array}{l}\text { Methyl glucosyl-3,4-dehydro-apo-8- } \\
\text { lycopenoate. }\end{array}$ & $\begin{array}{l}\text { Planococcus maritimus } \\
\text { МКИоо9 }\end{array}$ & Firmicutes & Pos (+) & $\begin{array}{l}\text { Suspended cell without lyophilizate in } \\
1.0 \mathrm{~N} \mathrm{NaOH}+\text { ultrasound } \times 5 \text { min + } \\
\text { centrifugation + DCM:MeOH for } 3 \text { times } \\
\text { + contrated + suspende EtOAc:water. }\end{array}$ & $\begin{array}{l}\text { Preparative silica gel HPLC }(20 \times 250 \\
\text { mm) with DCM:MeOH; preparative } \\
\text { ODS HPLC (20 x } 250 \mathrm{~mm}) \text { with } \\
\text { MeOH 96\%, APCI- HR-MS. }\end{array}$ & {$[85,86]$} \\
\hline $\begin{array}{lr}\text { all-E-sarcinaxanthin, } & \text { all-E- } \\
\text { decaprenoxanthin, decaprenoxanthin } \\
\text { mono- and diglucosides, } & \text { cis-9-Z- } \\
\text { decaprenoxanthin, } & \text { cis-15-Z- } \\
\text { decaprenoxanthin, } & \end{array}$ & Arthrobacter arilaitensis & Actinobacteria & Pos $(+)$ & $\begin{array}{l}\text { Lyophilised cells }+\mathrm{MeOH}+\text { ultrasound } \\
\text { x } 90 \text { min to r.t. }+ \text { centrifugation }+ \text { re- } \\
\text { extracted two times with } \mathrm{MeOH} \text { and } \\
\text { equally MTBE. }\end{array}$ & $\begin{array}{l}\text { HPLC-DAD-APCI-MS (IT-TOF): } \\
\text { C18 column } 2.7 \mu \mathrm{m}(4.6 \text { x } 150 \mathrm{~mm}) ; \\
\text { mobile phase: (A) MeOH, (B) MTBE. } \\
\text { Isocratic and gradient elution. }\end{array}$ & {$[18]$} \\
\hline
\end{tabular}




\begin{tabular}{|c|c|c|c|c|c|c|}
\hline hydroxyspirilloxanthin. & $\begin{array}{l}\text { Roseomonas aerofrigidensis } \\
\text { sp.nov. }\end{array}$ & Proteobacteria & Neg $(-)$ & Lyophilized cells $+\mathrm{MeOH}$ :acetone $(7: 2)$ & $\begin{array}{l}\text { HPLC-DAD: C18 column, mobile } \\
\text { phase: ACN:MeOH:AcEtOH (5:4:1). } \\
\text { Isocratic elution. }\end{array}$ & {$[87,88]$} \\
\hline $\begin{array}{l}\text { Spirilloxanthin, rhodopin, } \\
\text { anhydrorhodovibrin, tetrahydrolycopene, } \\
\text { rhodovibrin. }\end{array}$ & $\begin{array}{l}\text { Ectothiorhodospira salini } \\
\text { JA430T }\end{array}$ & Proteobacteria & Neg $(-)$ & Lyophilized cells + MeOH:acetone (7:2). & $\begin{array}{l}\text { HPLC-DAD: C18 column, mobile } \\
\text { phase: ACN:MeOH:AcEtOH (5:4:1). } \\
\text { Isocratic elution. }\end{array}$ & {$[88]$} \\
\hline $\begin{array}{l}\text { trans-Diadinoxanthine, cis- } \\
\text { diadinoxanthine, isomer diadinoxanthine, }\end{array}$ & Bacillus licheniformis & Firmicutes & Pos $(+)$ & $\begin{array}{l}\text { Cell without liofilizate }+ \text { cold } \\
\text { acetone:MeOH(7:3) }+ \text { ultrasound }+ \\
\text { centrifugate. }\end{array}$ & $\begin{array}{l}\text { HPLC-DAD: C18 column } 5 \mu \mathrm{m}(4 \mathrm{x} \\
250 \mathrm{~mm}) ; \quad \text { mobile phase: } \\
\text { ACN:MeOH. Isocratic elution. }\end{array}$ & [89] \\
\hline Decaprenoxanthin and analogous. & $\begin{array}{l}\text { Arthrobacter } \\
\text { psychrochitiniphilus } 366\end{array}$ & Actinobacteria & Pos $(+)$ & $\begin{array}{l}\text { Lyophilized cells }+\mathrm{MeOH}+\text { shake } 5 \mathrm{~min} \\
+ \text { concentration + hexane + shake } 10 \mathrm{~min} \\
+\mathrm{NaCl} \text { solution }(10 \%)+\text { shake + organic } \\
\text { layer collected + re-extracted } \\
\begin{array}{l}\text { hexane:diethyl ether } \quad(3: 1) \text { and } \\
\text { saponificate analysis. }\end{array}\end{array}$ & 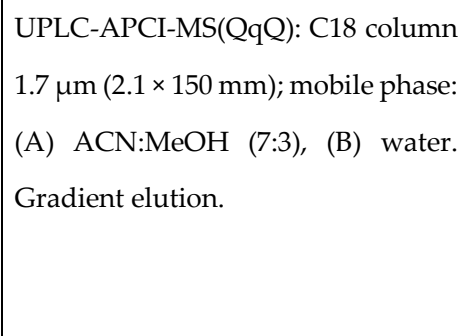 & [17] \\
\hline $\begin{array}{l}\text { Decaprenoxanthin, mono- and di- } \\
\text { glycosylated decaprenoxanthin. }\end{array}$ & Arthrobacter sp. M3 & Actinobacteria & Pos $(+)$ & $\begin{array}{l}\text { Cells without lyophilize } \quad+ \\
\text { MeOH:acetone }(1: 1) \quad+\text { dryness. }\end{array}$ & No Report & {$[33,90]$} \\
\hline $\begin{array}{l}\text { Saproxanthin, dehydroflexixanthin, } 20- \\
\text { Isopentenyldehydrosaproxanthin. }\end{array}$ & Rhodopirellula rubra $L F 2 T$ & Planctomycetes & $\operatorname{Neg}(-)$ & $\begin{array}{l}\text { Cells without lyophilizate }+ \text { solvent } \\
\text { extracts. }\end{array}$ & No Report & [14] \\
\hline $\begin{array}{l}\text { Saproxanthin, dehydroflexixanthin } 20- \\
\text { Isopentenyldehydrosaproxanthin. }\end{array}$ & $\begin{array}{l}\text { Rubinisphaera brasiliensis } \\
\text { Gr7 }\end{array}$ & Planctomycetes & $\operatorname{Neg}(-)$ & $\begin{array}{l}\text { Cells without lyophilizate }+ \text { solvent } \\
\text { extracts. }\end{array}$ & No Report & [14] \\
\hline $\begin{array}{l}\text { 3,4-didehydrorhodopin, } \quad \text { rhodopin, } \\
\text { rhodovibrin, } \\
\text { anhydrorlloxanthin, } \\
\end{array}$ & $\begin{array}{l}\text { Rhodopseudomonas palustris } \\
\text { CQV97 }\end{array}$ & Proteobacteria & $\operatorname{Neg}(-)$ & $\begin{array}{l}\text { Cell without lyophilizate }+ \\
\text { acetone:MeOH }(7: 2)+\text { ultrasound until } \\
\text { colorless. }\end{array}$ & $\begin{array}{l}\text { HPLC-ESI-MS(IT): C18 column (4.6 } \\
\text { x } 150 \mathrm{~mm}) ; \text { mobile phase: (A) } \\
\text { ACN:water (95:5), (B) MeOH. } \\
\text { Isocratic elution. }\end{array}$ & [91] \\
\hline
\end{tabular}




\begin{tabular}{|c|c|c|c|c|c|c|}
\hline $\begin{array}{l}\text { Hydroxydemethylspheroidene and } \\
\text { unkouwn carotenoid. }\end{array}$ & Hoeflea olei sp. nov. & Proteobacteria & $\operatorname{Neg}(-)$ & $\begin{array}{l}\text { Lyophilized cells + MeOH:acetone (7:2) } \\
+ \text { ultrasound + centrifugate + dryness. }\end{array}$ & $\begin{array}{l}\text { HPLC-DAD: C18 column }(4.6 \text { x } 250 \\
\text { mm); mobile phase: ACN:MeOH: } \\
\text { AcEtOH (5:4:1). Isocratic elution. } \\
\text { HPLC-ESI-MS: C18 column }(4.6 \times 50 \\
\text { mm); mobile phase: ACN:MeOH } \\
\text { (6:4). Isocratic elution. }\end{array}$ & {$[92,93]$} \\
\hline Spirilloxanthin & Afifella aestuarii sp. nov. & Proteobacteria & Neg (-) & $\begin{array}{l}\text { Lyophilized cells }+ \text { acetone:MeOH } \\
(9: 0.5)+\text { ultrasound }+ \text { centrifugation }+ \\
\text { dryness. }\end{array}$ & $\begin{array}{l}\text { HPLC-DAD: C18 column }(4.6 \times 250 \\
\text { mm); mobile phase: ACN:MeOH: } \\
\text { AcEtOH (5:4:1). Isocratic elution. } \\
\text { HPLC-ESI-MS: C18 column }(4.6 \times 50 \\
\text { mm); mobile phase: ACN:MeOH } \\
\text { (6:4). Isocratic elution. }\end{array}$ & {$[78,92]$} \\
\hline $\begin{array}{l}\left(2^{\prime} \text { S)-deoxymyxol } \quad 1^{\prime} \text {-glucoside } \quad\left(2^{\prime} \mathrm{S}\right)-4-\right. \\
\text { ketodeoxymyxol 1'-glucoside. }\end{array}$ & Gordonia terrae AIST-1 & Actinobacteria & Pos $(+)$ & $\begin{array}{l}\text { Lyophilized cells + acetone: } \\
\mathrm{MeOH}(7: 2)+\text { ultrasound }+ \\
\text { centrifugation }+ \text { organic solvent } \\
\text { evaporation. }\end{array}$ & $\begin{array}{l}\text { HPLC-DAD: C18 column }(8 \quad x \\
\text { 100mm); mobile phase: }(\mathrm{A}) \text { water }(\mathrm{B}) \\
\mathrm{CHCl}_{3} \text {. Gradient elution. }\end{array}$ & [94] \\
\hline Flexirubin-type & Chryseobacterium sp. kr6 & Bacteroidetes & Neg (-) & $\begin{array}{l}\text { Lyophilized cells + acetone + ultrasonic } \\
+ \text { centrifugation }+ \text { dried with } \mathrm{N}_{2} \text { (gas). }\end{array}$ & $\begin{array}{l}\text { HPLC-DAD-APCI-MS: C18 column } \\
(4.6 \times 250 \mathrm{~mm}) \text {; mobile phase: }(\mathrm{A}) \\
\text { water, (B) MeOH both with formic } \\
\text { acid }(0.1 \%) \text {. Gradient elution }\end{array}$ & [95] \\
\hline $\begin{array}{l}\text { Isorenieratene, 3-hydroxyisorenieratene, } \\
\text { 3,3'-dihydroxy-isorenieratene. }\end{array}$ & Brevibacterium linens & Actinobacteria & Pos $(+)$ & $\begin{array}{l}\text { Cell without lyophilize }+\mathrm{MeOH}+50 \\
\text { rpm agitation } \times 2 \text { hours }+ \text { centrifugation } \\
+\mathrm{Na}_{2} \mathrm{SO}_{4} \text { (Anh.) }+ \text { evaporation under } \\
\text { vacuum. }\end{array}$ & 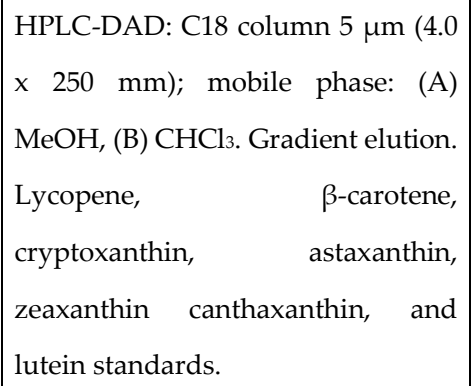 & [96] \\
\hline
\end{tabular}




\begin{tabular}{|c|c|c|c|c|c|c|}
\hline $\begin{array}{l}\text { Luteine, lycopenal, lycopenal glucoside, } \\
\text { rhodopin, rhodopinal, rhodopinol, } \\
\text { rhodopinglucoside. }\end{array}$ & $\begin{array}{l}\text { Rhodoblastus sphagnicola sp. } \\
\text { Nov }\end{array}$ & Proteobacteria & $\operatorname{Neg}(-)$ & $\begin{array}{l}\text { Cells without lyophilize }+ \\
\text { MeOH:acetone }(1: 1) \quad+\text { ultrasonid }+ \\
\text { centrifugate }+ \text { dryness. }\end{array}$ & $\begin{array}{l}\text { HPLC-DAD: C18 column } 5 \mu \mathrm{m}(4.0 \\
\mathrm{mm} \times 250 \mathrm{~mm} \text { ); mobile phase: (A) } \\
\text { ACN, (b) water (C) AcOEt. Gradient } \\
\text { elution }\end{array}$ & [97] \\
\hline $\begin{array}{l}\text { Canthaxanthin, phytoene, astaxanthin, } \\
\text { lycopene, phytofluene, } \\
\text { tetrahydrosqualene, } \\
\text { hydroxyechinenone, } \\
\text { bisanhydrobacterioruberin, } \\
\text { monoanhydrobacterioruberin, } \\
\text { bacterioruberin, haloxanthin. }\end{array}$ & \begin{tabular}{|l} 
Haloferax \\
alexandrinus $\quad$ GUSF-1 \\
(KF796625)
\end{tabular} & Euryachaeota & $\operatorname{Neg}(-)$ & $\begin{array}{l}\text { Cell-free supernatant }+ \text { cold EtOH }+ \\
\text { overnight at }-20^{\circ} \mathrm{C}+\text { centrigutation. }\end{array}$ & $\begin{array}{l}\text { HPLC-DAD-ESI-MS(QTOF): } \\
\text { UHPLC and Nano-HPLC; C-18 } \\
\text { column } 3 \mu \mathrm{m}(2.1 \times 100 \mathrm{~mm}) \text {. Mobile } \\
\text { phase: (A) water with } 0.1 \% \text { formic } \\
\text { acid (B) ACN:MeOH (7:3). }\end{array}$ & [98] \\
\hline $\begin{array}{l}\text { Astaxanthin, adonixanthin, hydroxy- } \\
\text { astaxanthin, dihydroxy-astaxanthin. }\end{array}$ & Brevundimonas sp. & Proteobacteria & $\operatorname{Neg}(-)$ & $\begin{array}{l}\text { Cell without lyophilizate suspended } \\
\mathrm{DMSO}+\text { shake at } 50^{\circ} \mathrm{C}+\mathrm{MeOH}+ \\
\text { centrifugation. }\end{array}$ & 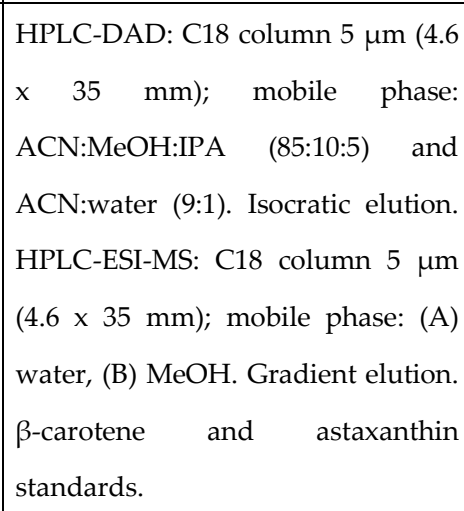 & {$[25,99-101]$} \\
\hline 2,2'-dihydroxy-astaxanthin, astaxanthin. & Brevundimonas scallop & Proteobacteria & $\operatorname{Neg}(-)$ & $\begin{array}{l}\text { Lyophilized cells }+ \text { acetone }+ \text { rotary } \\
\text { shaker }+ \text { centrifugation }+ \text { dried with } \mathrm{N}_{2} \\
\text { (gas) }\end{array}$ & $\begin{array}{l}\text { HPLC-DAD and HPLC-ESI-MS; } \\
\text { C18 column (Hypersil ODS); mobile } \\
\text { phase: MeOH:water (95:5). Isocratic } \\
\text { elution. }\end{array}$ & [102] \\
\hline
\end{tabular}




\begin{tabular}{|c|c|c|c|c|c|c|}
\hline Astaxanthin and astaxanthin isomers. & $\begin{array}{l}\text { Sphingomonas } \\
\text { astaxanthinifaciens }\end{array}$ & Proteobacteria & $\operatorname{Neg}(-)$ & $\begin{array}{l}\text { Cells without lyophilize }+\mathrm{MeOH}+ \\
\text { rotary shaker at } 50^{\circ} \mathrm{C}+\text { centrifugation }\end{array}$ & 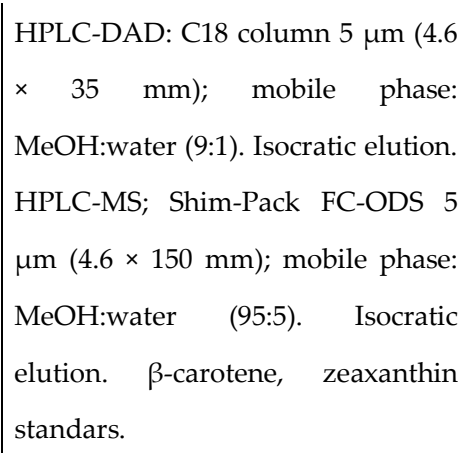 & [103] \\
\hline Astaxanthin and 4-ketozeaxanthin & Agrobacterium aurantiacum & Proteobacteria & $\operatorname{Neg}(-)$ & $\begin{array}{l}\text { Cells without lyophilize }+ \text { acetone }+ \\
\text { vacuum distillation }+\mathrm{AcOEt}^{-} \text {water }+ \\
\text { organic phase with } \mathrm{Na}_{2} \mathrm{SO}_{4} \text { (Anh.) }+ \\
\text { vacuum distillation. }\end{array}$ & 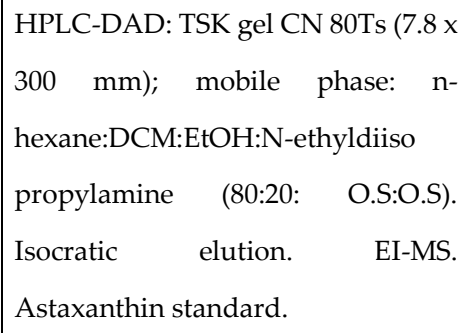 & [101] \\
\hline \multirow[t]{3}{*}{ Astaxanthin } & $\begin{array}{l}\text { Halobacillus trueperi MXM- } \\
16\end{array}$ & Firmicutes & Pos (+) & $\begin{array}{l}\text { Cell without lyophilizate }+ \\
\text { MeOH:acetone }(1: 1)+\text { ultrasound } \times 2 \\
\text { min }+ \text { centrifugation. }\end{array}$ & $\begin{array}{l}\text { HPLC-DAD: C18 column } 5 \mu \mathrm{m} \\
(4 \times 25 \mathrm{~mm}) \text {; mobile phase: } \mathrm{MeOH} \text {. } \\
\text { Isocratic elution. }\end{array}$ & {$[30]$} \\
\hline & Exiguobacterium Sps & Firmicutes & Pos (+) & $\begin{array}{l}\text { Cell pellet wash sterile water }+ \\
\text { suspended } 5 \mathrm{~mL} \mathrm{MeOH}+\text { incubated } \\
\text { water bath at } 60{ }^{\circ} \mathrm{C} \times 15 \mathrm{~min}+ \\
\text { centrifugation }\end{array}$ & $\begin{array}{l}\text { HPLC-UV-vis: C18 column (4.6 x } \\
250 \mathrm{~mm} \text { ); mobile phase: (A) water, } \\
\text { (B) MeOH. Gradient elution. }\end{array}$ & [31] \\
\hline & Sphingomonas faeni ISY & Proteobacteria & $\operatorname{Neg}(-)$ & $\begin{array}{l}\text { Cells without lyophilize }+\mathrm{sequential} \\
\text { extraction with hexane, } \mathrm{CHCl}_{3} \text { and } \\
\mathrm{MeOH}+\text { overnightin an orbital shaker }+ \\
\text { re-extraction }+ \text { centrifugation for } 15 \mathrm{~min} \\
\text { at } 4^{\circ} \mathrm{C}+\text { solvent evaporation under } \\
\text { vacuum. }\end{array}$ & $\begin{array}{l}\text { HPLC-UV-vis: C18 column; mobile } \\
\text { phase: acetone:MeOH:THF } \\
\text { (70:28:2). Isocratic elution. HPLC- } \\
\text { MS(QqQ): C18 column; mobile } \\
\text { phase: water (A) and ACN (B) both } \\
\text { with } 0.1 \% \text { formic acid. Gradient } \\
\text { elution. }\end{array}$ & [104] \\
\hline
\end{tabular}




\begin{tabular}{|c|c|c|c|c|c|c|}
\hline Astaxanthin, cantaxanthin, lutein. & $\begin{array}{l}\text { Gordonia alkanivorans strain } \\
1 B\end{array}$ & Actinobacteria & Pos $(+)$ & $\begin{array}{l}\text { Cells dried at } 55^{\circ} \mathrm{C} \times 1 \mathrm{~h}+\text { DMSO }+ \\
\text { orbital shaker at } 50{ }^{\circ} \mathrm{C}+\text { centrifugate }+ \\
\text { repeated until colorless + acetone: } \mathrm{NaCl} \\
\text { solution }(20 \%): \mathrm{AcEtOH}+\text { concentration } \\
\text { phase AcEtOH. }\end{array}$ & 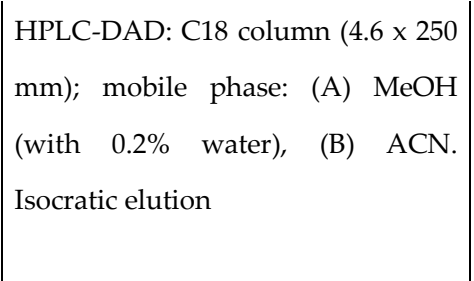 & [105] \\
\hline Astaxanthin and canthaxanthin. & Methylomonas sp. $16 a$ & Proteobacteria & $\operatorname{Neg}(-)$ & $\begin{array}{l}\text { Cells without lyophilize }+ \text { glass beads }+ \\
\text { EtOH }+ \text { vortex }+ \text { DCM }+ \text { vortex }+ \\
\text { centrifuge and dried. }\end{array}$ & 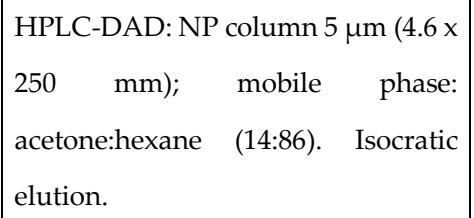 & {$[75,106]$} \\
\hline Cantaxanthin & Bradyrhizobium sp. & Proteobacteria & $\operatorname{Neg}(-)$ & $\begin{array}{l}\text { Cells without lyophilize }+ \\
\text { MeOH:acetone }(7: 2)+\text { solution } \mathrm{NaCl} \\
(10 \%)+\text { hexane + organic phase vacuum } \\
\text { distillation. }\end{array}$ & 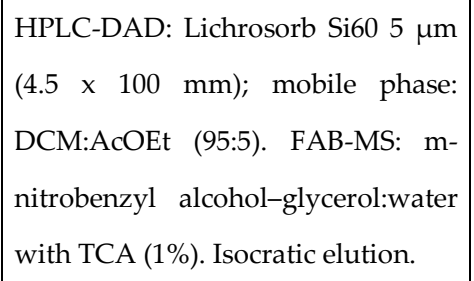 & [107] \\
\hline Canthaxanthin isomers & Dietzia sp. & Actinobacteria & Pos $(+)$ & $\begin{array}{l}\text { Lyophilized cells }+\mathrm{CHCl}_{3}: \mathrm{MeOH}(1: 1)+ \\
\text { rotavapor + resuspended in cold acetone } \\
+ \text { centrifugation }+ \text { stored at }-20^{\circ} \mathrm{C} \text { with } \\
\mathrm{N}_{2} \text { (gas). }\end{array}$ & $\begin{array}{l}\text { HPLC-DAD, HPLC-APCI-MS/MS: } \\
\text { C18 column }(4.6 \times 150 \mathrm{~mm}) ; \text { mobile } \\
\text { phase: ACN:MeOH (7:3); Isocratic } \\
\text { elution. Astaxanthin, } \beta \text {-carotene and } \\
\text { all-trans-canthaxanthin standards. }\end{array}$ & [108] \\
\hline $\begin{array}{l}\text { Zeaxanthin, } \beta \text {-cryptoxanthin, } \beta \text {-carotene, } \\
\text { phytoene. }\end{array}$ & Zobellia laminarie 465 & Bacteroidetes & $\operatorname{Neg}(-)$ & $\begin{array}{l}\text { Lyophilized cells }+\mathrm{MeOH}+\text { shake } 5 \mathrm{~min} \\
+ \text { hexane with } 10 \text { min shake }+ \text { solution } \\
\mathrm{NaCl}(10 \%)+\text { shake }+ \text { organic layer } \\
\text { collected }+ \text { re-extraction hexane:diethyl } \\
\text { ether }(3: 1) \text { and saponificate analysis. }\end{array}$ & $\begin{array}{l}\text { HPLC-DAD: C18 column } 1.7 \mu \mathrm{m} \\
(2.1 \times 150 \mathrm{~mm}) \text {; mobile phase: }(\mathrm{A}) \\
\text { ACN, } \quad \text { (B) } \mathrm{MeOH}, \quad \text { (C) } \text { water. } \\
\text { Gradient elution. }\end{array}$ & [17] \\
\hline $\begin{array}{l}\text { Zeaxanthin, } \quad \text { cryptoxanthin, } \\
\text { myxoxanthophyll. }\end{array}$ & $\begin{array}{l}\text { Synechococcus sp. strain } \\
\text { PCC } 7002\end{array}$ & Synechococcaceae & $\operatorname{Neg}(-)$ & $\begin{array}{l}\text { Cell without lyophilizate }+ \\
\text { acetone:MeOH }(7: 2) \text { or pure } \mathrm{MeOH}+ \\
\text { ultrasound }+ \text { centrifugation. }\end{array}$ & $\begin{array}{l}\text { HPLC-DAD: C18 column } 5 \mu \mathrm{m} \text { (4.6 } \\
\text { mm x } 250 \mathrm{~mm} \text { ); ACN:MeOH:water } \\
\text { (with } 10 \mathrm{mM} \mathrm{NH} \mathrm{NHAc}_{4} \text { ). }\end{array}$ & {$[44,109]$} \\
\hline
\end{tabular}




\begin{tabular}{|c|c|c|c|c|c|c|}
\hline \multirow[t]{5}{*}{ Zeaxanthin } & $\begin{array}{l}\text { Mesoflavibacter } \\
\text { zeaxanthinifaciens }\end{array}$ & Bacteroidetes & $\operatorname{Neg}(-)$ & $\begin{array}{l}\text { Cells without lyophilize }+\mathrm{MeOH}+ \\
\text { rotary shaker at } 50^{\circ} \mathrm{C}+\text { solution } \mathrm{NaCl} \\
(10 \%)+\text { hexane }+ \text { organic phase vacuum } \\
\text { distillation. }\end{array}$ & 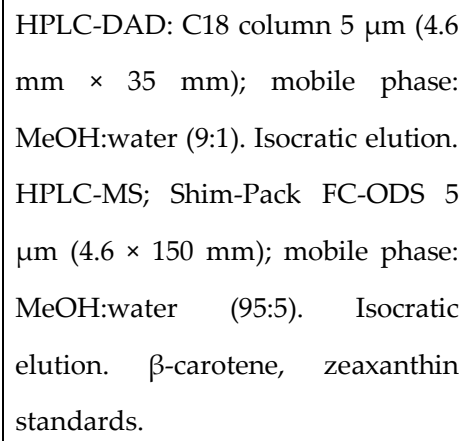 & [110] \\
\hline & Formosa sp. KMW & Bacteroides & Neg (-) & $\begin{array}{l}\text { Cells suspended in sterile saline + } \\
\text { lysozyme }(20 \mathrm{mg} / \mathrm{ml})+\text { acetone }+ \text { Filter }+ \\
\text { hexane }+\mathrm{Na}_{2} \mathrm{SO}_{4} \text { (Anh) }\end{array}$ & $\begin{array}{l}\text { HPLC-DAD: C18 column }(4.6 \times 250 \\
\text { mm); mobile phase: (A)MeOH, } \\
\text { (B)water, (C) THF. Gradient elution. }\end{array}$ & [65] \\
\hline & Vitellibacter sp. NMW & Bacteroides & $\operatorname{Neg}(-)$ & $\begin{array}{l}\text { Cells suspended in sterile saline }+ \\
\text { lysozyme }(20 \mathrm{mg} / \mathrm{ml})+\text { acetone }+ \text { filter }+ \\
\text { hexane }+\mathrm{Na}_{2} \mathrm{SO}_{4} \text { (Anh.). }\end{array}$ & $\begin{array}{l}\text { HPLC-DAD: C18 column }(4.6 \times 250 \\
\text { mm); mobile phase: (A)MeOH, } \\
\text { (B)water, (C) THF. Gradient elution. }\end{array}$ & [65] \\
\hline & $\begin{array}{l}\text { Synechococcus elongatus } \\
\text { PCC } 7942\end{array}$ & Cyanobacteria & Neg $(-)$ & $\begin{array}{l}\text { Dry cell }+\mathrm{MeOH}+\text { centrifugation }+ \text { re- } \\
\text { suspended } \mathrm{MeOH}+\text { sonication }+ \\
\text { centrifugation. }\end{array}$ & 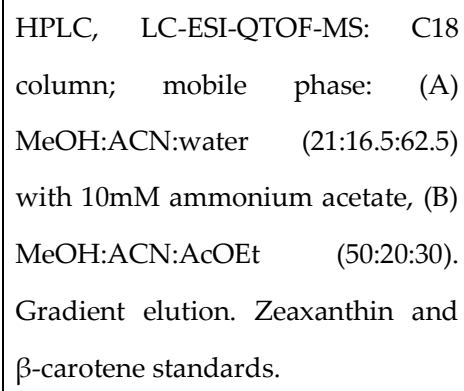 & [111] \\
\hline & $\begin{array}{l}\text { Paracoccus } \\
\text { zeaxanthinifaciens ATCC } \\
21588\end{array}$ & Proteobacteria & $\operatorname{Neg}(-)$ & $\begin{array}{l}\text { Cell pellet wash sterile water }+\mathrm{THF}+ \\
\text { centrifugation. }\end{array}$ & $\begin{array}{l}\text { HPLC-DAD: C } 30 \text { column } 5 \mu \mathrm{m}(4.6 \\
\text { x } 250 \mathrm{~mm} \text { ) with a C18 guard column } \\
(20 \mathrm{~mm}) \text {; mobile phase: (A) MeOH, } \\
\text { (B) MTBE (8:2). Gradient elution. } \\
\text { Astaxanthin, 13-cis-zeaxanthin, 15- } \\
\text { cis-zeaxanthin, all-trans-zeaxanthin, }\end{array}$ & [112] \\
\hline
\end{tabular}




\begin{tabular}{|c|c|c|c|c|c|c|}
\hline & & & & & $\begin{array}{l}\text { canthaxanthin, cryptoxanthin, } \beta- \\
\text { carotene, and lycopene standards. }\end{array}$ & \\
\hline $\begin{array}{l}\text { Lycopene, } \quad \beta \text {-carotene, } \quad \text { zeaxanthin, } \\
\text { canthaxanthin, astaxanthin. }\end{array}$ & $\begin{array}{l}\text { Corynebacterium } \\
\text { glutamicum }\end{array}$ & Actinobacteria & Pos $(+)$ & $\begin{array}{l}\text { Cell resuspende }+\mathrm{MeOH} \text { :acetone (7:3) } \\
\text { with } 0.05 \% \mathrm{BHT} \text { at } 60{ }^{\circ} \mathrm{C} \times 15 \mathrm{~min} \text { with } \\
\text { vortex every } 5 \mathrm{~min}+\text { centrifugation. }\end{array}$ & 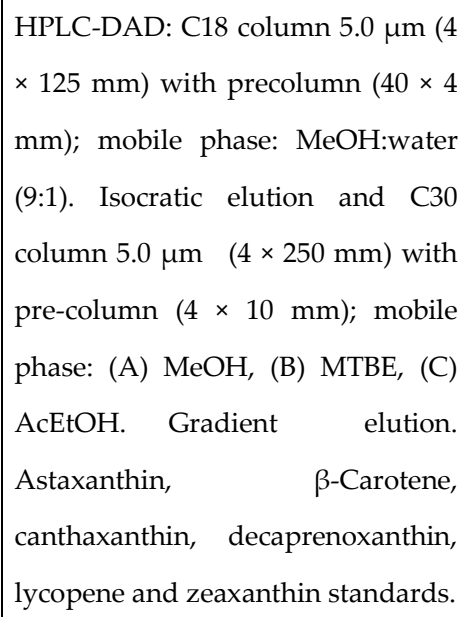 & [66] \\
\hline Adonixanthin & Erythrobacter & Proteobacteria & Neg (-) & $\begin{array}{l}\text { Cell without lyophilizate }+ \text { DMSO }+ \\
\text { Shake at } 50{ }^{\circ} \mathrm{C}+\mathrm{MeOH}+\text { vortex }+ \\
\text { centrifugation }\end{array}$ & $\begin{array}{l}\text { HPLC-DAD: C18 column } 5 \mu \mathrm{m}(4.6 \\
x \quad 35 \mathrm{~mm}) ; \quad \text { mobile phase: } \\
\text { ACN:MeOH:THF (5.8:3.5:0.7) and } \\
\text { ACN:water (9:1). Isocratic elution. } \\
\text { LC-ESI-MS: Shim-Pack FC-ODS } 5 \\
\mu \mathrm{m}(4.6 \text { x } 150 \mathrm{~mm}) \text {; mobile phase: } \\
\text { (A) MeOH (B) water (95:5). Gradient } \\
\text { elution. } \beta \text {-carotene, astaxanthin, } \\
\text { zeaxanthin, canthaxanthin, and } \\
\text { echinenone standards. }\end{array}$ & [13] \\
\hline $\begin{array}{l}\text { Neurosporene, } \alpha \text {-carotene, canthaxanthin, } \\
\text { astaxanthin, } \quad \beta \text {-carotene, neoxanthin, } \\
\text { zeaxanthin, phytofluene. }\end{array}$ & Asaia lannensis & Proteobacteria & Neg (-) & $\begin{array}{l}\text { Cell without lyophilizate }+ \text { wash PBS } \\
\text { solution + acetone: } \mathrm{MeOH}(7: 3)+\text { shake in } \\
\text { darkness at } 60^{\circ} \mathrm{C} \times 2 \text { hours + } \\
\text { centrifugation } 6500 \mathrm{rpm} \times 10 \mathrm{~min} .\end{array}$ & $\begin{array}{l}\text { HPLC-DAD-ESI-MS(Orbitrap): C30 } \\
\text { column } 2.6 \mu \mathrm{m}(3.0 \times 100 \mathrm{~mm}) ; \\
\text { Mobile phase: (A) water, (B) MeOH, } \\
\text { (C) MTBE. Gradient elution. }\end{array}$ & [113] \\
\hline
\end{tabular}




\begin{tabular}{|c|c|c|c|c|c|c|}
\hline & Asaia bogorensis & Proteobacteria & $\operatorname{Neg}(-)$ & $\begin{array}{l}\text { Cell without lyophilizate }+ \text { wash PBS } \\
\text { solution + acetone:MeOH (7:3)+ shake in } \\
\text { darkness at } 60^{\circ} \mathrm{C} \text { × } 2 \text { hours + } \\
\text { centrifugation } 6500 \mathrm{rpm} \times 10 \mathrm{~min} .\end{array}$ & $\begin{array}{l}\text { HPLC-DAD-ESI- MS(Orbitrap): C30 } \\
\text { column } 2.6 \mu \mathrm{m}(3.0 \times 100 \mathrm{~mm}) \text {; } \\
\text { mobile phase: (A) water, (B) MeOH, } \\
\text { (C) MTBE. Gradient elution. }\end{array}$ & {$[113]$} \\
\hline \multirow[t]{2}{*}{ Neurosporene } & Kocuria sp.QWT-12 & Actinobacteria & Pos $(+)$ & $\begin{array}{l}\text { Cells washed sterile water }+\mathrm{MeOH}+ \\
\text { incubated water bath at } 60^{\circ} \mathrm{C} \times 15 \mathrm{~min} \\
\text { until colorless + filter. }\end{array}$ & No Report & [72] \\
\hline & Rhodobacter viridis JA737 & Proteobacteria & $\operatorname{Neg}(-)$ & $\begin{array}{l}\text { Dry cell mass + acetone:MeOH }(9: 0.5)+ \\
\text { sonication + centrifugation + solvent } \\
\text { evaporation with rotary evaporator. }\end{array}$ & $\begin{array}{l}\text { HPLC-DAD; } 18 \text { column }(4.6 \times 250 \\
\text { mm); mobile phase: } \\
\text { ACN:MeOH:AcOEt (5:4:1). Isocratic } \\
\text { elution. HPLC-ESI-MS; C18 } \\
\text { column }(50 \times 4.6 \mathrm{~mm}) \text {; mobile phase: } \\
\text { ACN:MeOH (6:4). Isocratic elution. }\end{array}$ & [92] \\
\hline $\begin{array}{l}\beta \text {-carotene, zeaxanthin, astaxanthin, } \\
\text { keto-myxocoxanthin glucoside-ester, } \\
\text { diapolycopenedioic-acid-diglu- cosyl- } \\
\text { ester. }\end{array}$ & $\begin{array}{l}\text { Exiguobacterium acetylicum } \\
\text { S01 }\end{array}$ & Firmicutes & Pos $(+)$ & $\begin{array}{l}\text { Cells without lyophilize }+ \text { mortar and } \\
\text { pestle }+\mathrm{MeOH} \text { :acetone }(7: 3)+ \\
\text { centrifugation }+ \text { organic solvent } \\
\text { evaporation. }\end{array}$ & 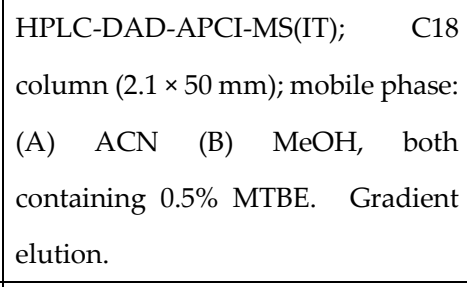 & {$[39,114,115]$} \\
\hline$\beta$-cryptoxanthin, $\beta$-carotene & Pseudomonas sp. & Proteobacteria & Neg (-) & $\begin{array}{l}\text { Cell without lyophilize }+\mathrm{MeOH}+\text { tris- } \\
\mathrm{HCl} \text { solution }(50 \mathrm{mM}, \mathrm{pH}=7.5)+\mathrm{NaCl} \\
\text { solution }(1 \mathrm{M})+\mathrm{CHCl}_{3}+\text { mixed } \times 5 \text { min } \\
+ \text { centrifugation }+ \text { lower phase } \\
\text { evaporation. }\end{array}$ & 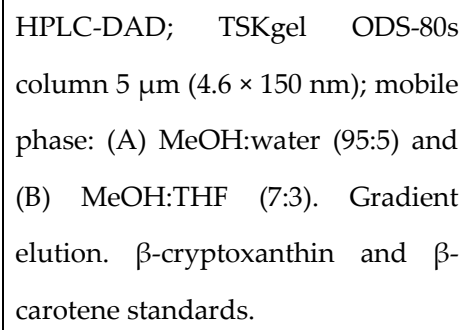 & {$[116]$} \\
\hline
\end{tabular}

The different solvents correspond to volume-volume ratios. Ahn.: anhydrous, ACN: acetonitrile, THF: tretrahydrofuran, MTBE: methyl tert-butyl ether IPA: isopropanol and HRMS: high-resolution mass spectrometry. 


\section{Characterization}

The complex carotenoid extract from bacteria needs to be separated before the different compounds can be identified. Hence, thin layer chromatography (TLC) and open column chromatography (OCC) have been traditionally employed for this separation step. These techniques involve off-line carotenoid separation frequently followed by mass spectrometric identification, as will be discussed in the next section. The major drawbacks of these techniques are the high sample concentration required and their low resolution, which curtails the ability to separate the wide variety of chemical structures reported for carotenoids and their intermediates (>1200 compounds). This complexity includes isomers (cis or trans), or modifications in the main chain that occur as a result of cyclization, addition of side chains, oxidation, hydrogenation, or dehydrogenation. Many of these structures are usually present in the bacterial carotenoid extract, making both their separation and identification very challenging. In addition, not all the carotenoid standards or their degradation products are available, which causes additional limitations to their identification [2].

Currently, the technique of choice to face all these analytical challenges is highperformance liquid chromatography (HPLC), usually coupled to a diode array detector (DAD) (or UV-visible detector) and mass spectrometry (MS). Therefore, the following paragraphs will discuss the HPLC separation methods reported on bacterial carotenoid extracts and analysis by DAD and MS.

\subsection{Chromatographic separation}

HPLC may use different types of columns available on the market such as cyano $(\mathrm{CN})$, octylsilane (C8), octadecylsilane (C18) or other more specific columns for separation of carotenoids (and some isomers) such as C30 [52]. Several reports have used C18 stationary phase columns in the analysis of bacterial carotenoids (see Table 1). The drawback of C18 columns is the poor resolution in the separation of cis/trans isomers of carotenes with 40 or more carbon atoms. However, when xanthophylls are analyzed, similar profiles are observed using C18 and C30 columns [2,117]. Features such as better stability at a high $\mathrm{pH}$, higher speed and low cost make the $\mathrm{C} 18$ column useful and convenient for simple carotenoid analysis compared to the C30 column [118]. C18 is also useful for other types of separation (vide infra). However, the higher retention capacity of both polar and non-polar compounds in C30 columns allows for a greater separation of carotenoids, thus favouring the resolution of geometric isomers, which is highly useful in complex carotenoid mixtures. Furthermore, $\mathrm{C} 30$ columns are stable at high $\mathrm{pH}$ and are more resistant to phase collapse than C18 columns. However, C30 columns generally require much longer analysis time than $\mathrm{C} 18$ columns.

Recently, Turcsi et al. [119] compared the separation capacity of C18 and C30 stationary phases using a 100 carotenoids sample divided into six main groups: carotenes, hydroxycarotenoids, epoxycarotenoids, ketocarotenoids, and reduced $\kappa$ carotenoids. The elution order of carotenoids in C18 columns was according to the polarity of each compound, with hydroxycarotenoids as the first eluted compounds, followed by carotenes, keto- and epoxy-carotenoids, which showed longer retention times. Similarly, the length of the molecule and the presence/number of polyenes are proportional to the retention times in C30 phases. Thus, the separation of regioisomers from compounds such as zeaxanthin and lutein, including their epoxides, is best achieved with the C 30 stationary phase. In contrast, $\mathrm{C} 18$ columns are efficient at separating polar carotenoid stereoisomers and epimers. Therefore, when analysing an unknown extract, the initial analysis with C18 phase is recommended, and the characterization is complemented using a C30 phase [117].

Another example of the versatility of the $\mathrm{C} 18$ stationary phase in ultra-high-pressure liquid chromatography with a diode array detector (UHPLC-DAD) was reported for the separation and quantification of 13 carotenoids including three phytoene isomers, twocarotene isomers, and two astaxanthin isomers [120]. Analysis was performed in a BEH 
C18 column and a Cortecs $\mathrm{C} 18$ column $(2.1 \times 50 \mathrm{~mm})$ at $35^{\circ} \mathrm{C}$ with a flow rate of $400 \mu \mathrm{l} / \mathrm{min}$, employing $\mathrm{MeOH}$ and $\mathrm{ACN}$ as mobile phase, with relatively short analysis times of 9 and 12 minutes. The method employed for both columns demonstrated a high level of reproducibility. However, the BEH C18 $(1.7 \mu \mathrm{m})$ showed a slightly higher performance than Cortecs C18 $(1.6 \mu \mathrm{m})$ in terms of recovery and less precision for the analysed carotenoids. Selectivity of the chromatographic method was demonstrated by analysing a carotenoid extract from $\mathrm{H}$. pluvialis. Thus, this method could be applied not only to algae samples, but also to bacteria. Moreover, with the goal of producing a fast and efficient chemical identification method of these bacterial metabolites, Asker's group and others have developed robust of screening and profiling methods for the simultaneous analysis of diverse strains of bacteria producing carotenoids, which has emerged as an efficient alternative $[13,99]$. Meanwhile, a HPLC-MS/MS method for the analysis of microbial carotenoids and quinones produced by five bacteria (Brevibacterium linens, Micrococcus luteus, Panthoea agglomerans, Rhodococcus equi, Arthrobacter bergerei, and Arthrobacter protophormiae) used a C18 column. This method allowed the structural elucidation of acyclic carotenes, mono and bicyclo carotenes, mono and bicyclo xanthophylls and acyclic xanthophylls [121].

Other reports have indicated the versatility of C8 columns in the separation of carotenoids. Thus, depending on the complexity of the carotenoid mixture, C8 columns have the advantage of shorter retention times compared to C18 and C30 columns; generally, C8 columns take around 20 minutes less than C18 and C30 columns [83,84,122]. Thus, it was possible to analyse fucoxanthinol, fucoxanthin, mactraxanthin, violaxanthin, neoxanthin and auroxanthin. It should be pointed out that the latter was an isomer of violaxanthin at acidic $\mathrm{pH}$ [122].

In 2017, Abate-Pella et al. [123] reported the use of a CN column in the efficient separation of more than 11 carotenoid standards in 9 or 18 minutes using $\mathrm{MeOH}$ as organic solvent. These cartenoids included carotene isomers ( $\alpha$-carotene, $\beta$-carotene and lycopene) and xanthophyll isomers (canthaxanthin, $\alpha$-cryptoxanthin and $\beta$ cryptoxanthin). The applicability of this chromatographic method was demonstrated on three biological samples of strawberry leaf, chicken feed supplement, and the bacterium Chloroflexus aurantiacus. The resolution factor was 0.75 for the three carotenoids present in this bacterium. In addition, cis/trans isomerization of $\alpha$-carotene was observed in the form of two peaks in the chromatogram for this carotene.

In summary, HPLC is the (gold) standard technique for carotenoid separation. However, it is worth noting that carotenoids are influenced by the physical properties of the solvents used. Therefore, the selection of a suitable solvent in the mobile phase can lead to drastic changes in the chromatographic separation. For this reason, $\mathrm{ACN}, \mathrm{MeOH}$, $\mathrm{THF}, \mathrm{CHCl}_{3}$, d-water, among other solvents, have been used (Table 1). In recent years, there has been an increase in the report of MeOH:MTBE:water mixtures as mobile phase with modifiers, such as ammonium acetate, ammonium formiate, acetic acid or formic acid, among others $[67,117,124-127]$, since their performances in the separation and identification of carotenoids has worked well (Figure 4).

However, many studies do not report the quantification of all carotenoids present in a sample because of the complexity of carotenoid chromatograms due to the high variety of isomers and structurally related compounds. In many cases, the standards of the carotenoids that are being analysed are not available, or only one of the isomers is available. Carotenoid quantification requires the use of the specific standard. Alternatively, when this is not possible, a different carotenoid, typically $\beta$-carotene is employed [128]. Even if chromatographic separation is achieved, the lack of standards still makes difficult to characterize this family of compounds. Mass spectrometry emerges as a useful technique for the identification and quantification of carotenoids. 


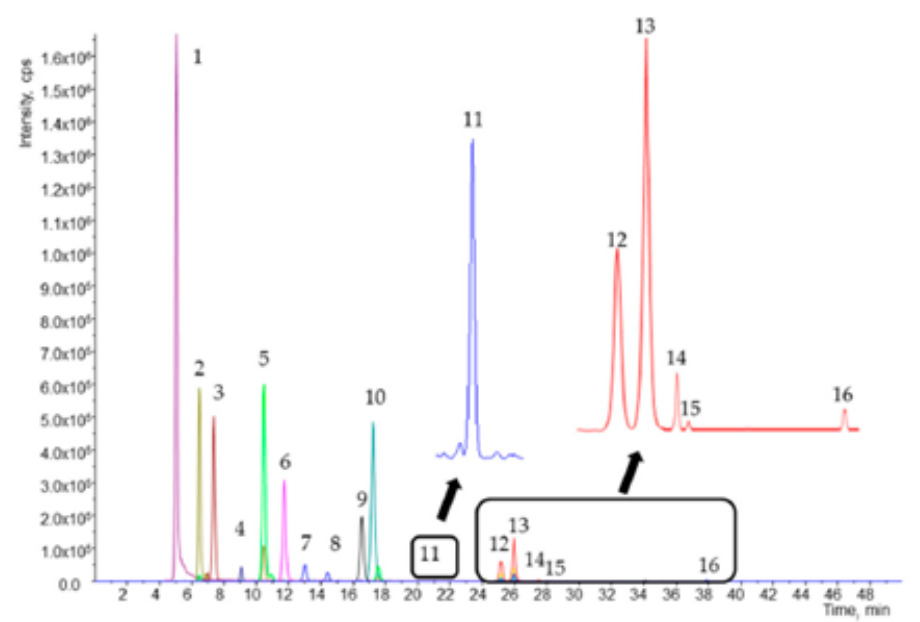

\author{
Compounds: \\ (1) retinol \\ (2) 25-hydroxycholecalciferol \\ (3) retinol acetate \\ (4) $a$-tocotriend \\ (5) cholecalciferol \\ (6) astaxanthin \\ (7) lutein \\ (8) zeaxanthin \\ (9) cantaxanthin \\ (10) E- $\beta$-apo-8'-carotenal \\ (11) cryptoxanthin \\ (12) 13-Z- $\beta$-carotene \\ (13) $\alpha$-carotene \\ (14) $\beta$-carotene \\ (15) 9 -Z- $\beta$-carotene \\ (16) 5-Z-lycopene.
}

Figure 4. Chromatographic separation of mixed standards of carotenes and xanthophylls on a C30 column using the mixture MeOH:MTBE:water with ammonium acetate. Adapted from Ref. [124].

\title{
4.2. Mass spectrometry analysis
}

Mass spectrometry (MS) provides structural information of the target compound though the molecular mass and fragmentation patterns that can be obtained in tandem mass spectrometry (MS/MS) mode [2,56]. MS selectivity allows the identification of compounds that cannot be separated by HPLC due to coelution. Therefore, HPLC-MS overcomes some of the problems related to the use of HPLC-DAD or HPLC-UV-vis for the identification of carotenoids since different families of these compounds have the same absorption in the UV-visible region.

In early studies, the identification of carotenoids was carried out using ionization methods such as electron ionization (EI), negative ion chemical ionization (CI) and fast atom bombardment (FAB) [56,129-131]. The main drawback of EI and CI methods is the carotenoids thermolability. These ionization sources require solvent removal at relatively high temperatures prior to compounds ionization. Although FAB was also implemented for LC-MS analysis of carotenoids, this ionization technique presents low ionization capacity in negative mode $[129,131,132]$. In addition, matrix-assisted laser desorption ionization (MALDI) has also been used in the identification of carotenoids [133-135]; unfortunately, MALDI does not allow the hyphenation of HPLC and MS; thus, complex samples need to be previously fractionated off-line. For example, Yoshida et al. used TLC for the separation of carotenoids from Bacillus subtilis followed by MALDI-TOF MS [136]. Similarly, off-line identification of carotenoids in Haloferax mediterranei was achieved using TLC-MALDI-TOF MS [137]. In addition, Schöner et al. [134] reported the characterization of bacterial carotenoids using preparative HPLC separation and identification by MALDI-TOF MS.

Two main ionization sources are currently used for carotenoid analysis: electrospray ionization (ESI) and atmospheric pressure chemical ionization (APCI). While ESI is useful in the analysis of ionic and polar compounds such as xanthophylls [117,138], APCI allows the characterization of both, carotenes and xanthophylls [117,132]. In recent years, a new ionization source, which is suitable for non-polar compounds, has been introduced. This source is known as atmospheric pressure photoionization (APPI) [139]. The ionization of metabolites in APPI is improved with the use of dopants; however, there is no universal solvent for this source, and the technique is still under development $[117,139]$.

In APCI it is possible to use mobile phases without buffers and with high flow rates (greater than $800 \mu \mathrm{L} / \mathrm{min}$ ), while ESI depends on the use of buffers or organic modifiers for efficient ionization and mobile phase flow rates lower or equal to $500 \mu \mathrm{L} / \mathrm{min}$. In addition, ESI, and $\mathrm{NaOH}$ solutions $(0.5 \mathrm{mmol} / \mathrm{L})$ have been used to increase the $\mathrm{Na}^{+}$ adducts of carotenoids, thus, improving the sensitivity of this ionization source [64]. 
Another characteristic of APCI is the possibility of distinguishing some isomeric carotenoids by comparison of the fragmentation patterns between the positive and negative modes $[132,139]$. Thus, the best ionization source for the identification of carotenes and xanthophylls is still APCI. Examples of positive and negative ionization in APCI are presented in Figures 5 and 6, showing the different sensitivity and selectivity that these two ionization processes can provide to the carotenoids analysis.

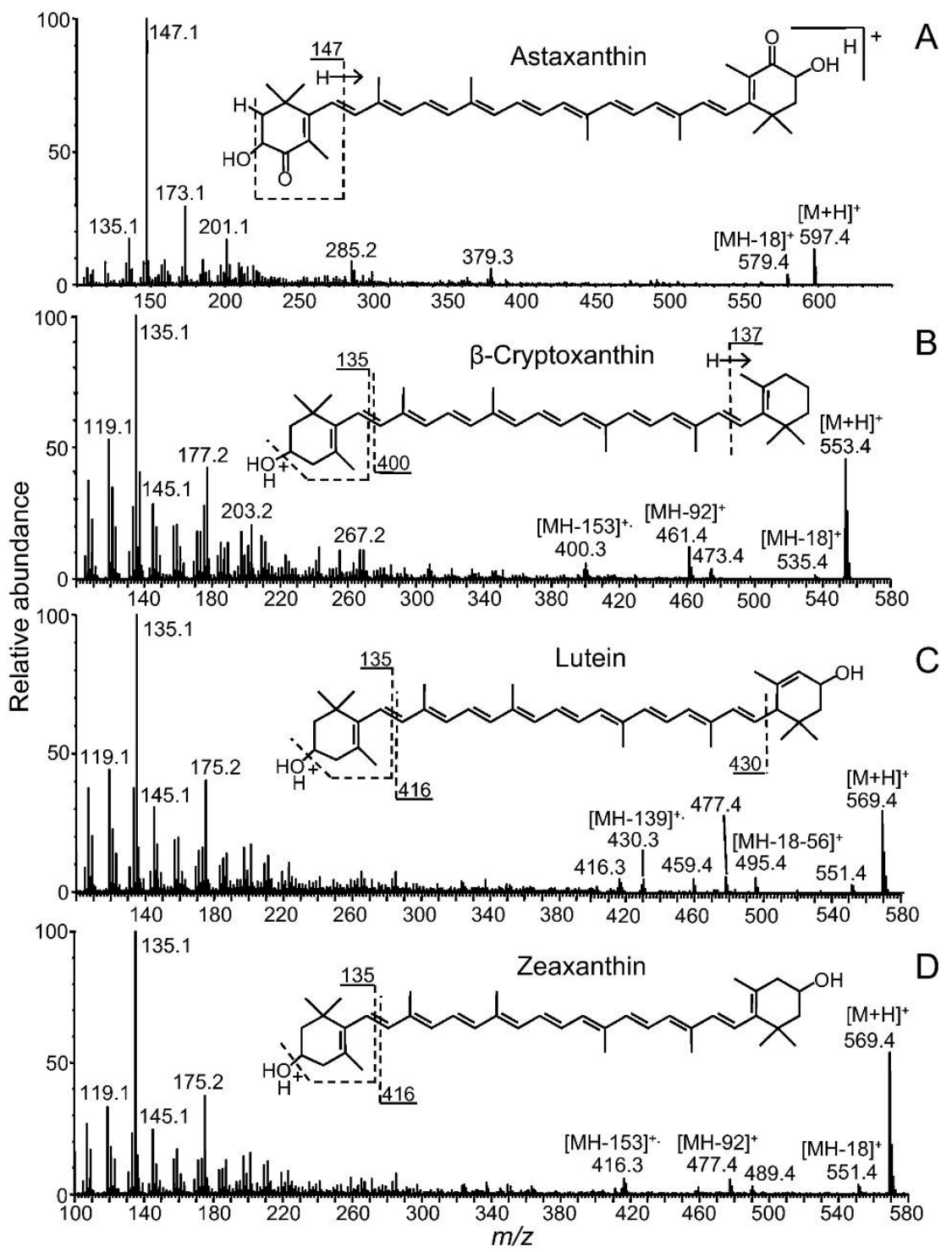

Figure 5. MS/MS spectra of xanthophylls in positive APCI: (A) astaxanthin; (B) $\beta$-cryptoxanthin; (C) lutein; and (D) zeaxanthin. Reprint from Ref. [132], with permission of Elsevier.

A comparative study between the three sources of ionization at atmospheric pressure (ESI, APCI and APPI) showed that the most powerful technique for carotenoids analysis is APCI [139]. In this study, 11 xanthophylls and four carotenes were analysed, and the effect of four dopants in APPI was evaluated. Carotenoids and carotenoid precursors observed with the strongest signal in APCI were: phytofluene, phytoene, echinenone, neoxanthin, antheraxanthin, astaxanthin, adonixanthin, zeaxanthin, $\beta$-apo-8'-carotenal, 3 hydroxyechinenone, $\beta$ - and $\alpha$-cryptoxanthin. ESI presented the best results for violaxanthin and lutein analysis, whereas APPI exhibited the highest level of ionization for lycopene and $\beta$-carotene. In addition, the four dopants employed (acetone, toluene, anisole, and chlorobenzene) showed increases in the signal for all carotenoids analysed in APPI. As a result, the highest sensitivity effect of dopants was observed for carotenes. 
Also, the addition of organic modifiers such as ammonium acetate in the mobile phase has been reported as a determining factor to increasing the ionization yield when using the APCI source [121].

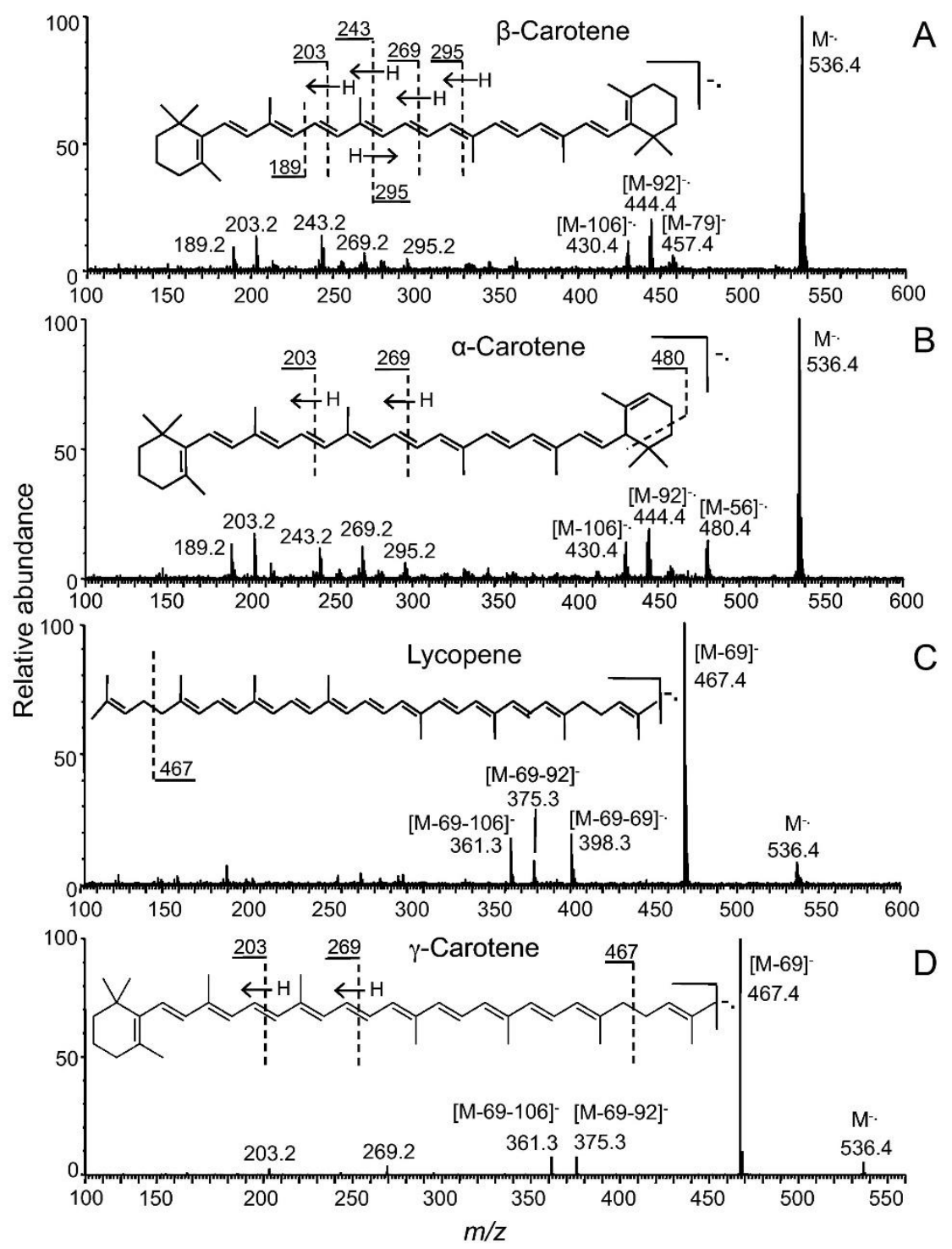

Figure 6. MS/MS spectra of carotenes in negative APCI: (A) $\beta$-carotene; (B) $\alpha$-carotene; (C) lycopene; and (D) $\gamma$-carotene. Reprint from Ref. [132], with permission of Elsevier.

Once these metabolites are separated and ionized, they pass to the mass analyser. Four types of analysers are mainly used for the identification of carotenoids from bacteria (see Table 1): ion trap (IT), triple quadrupole (QqQ), quadrupole time of flight (QTOF) and Orbitrap. IT allows the structural elucidation of unknown carotenoids by successive fragmentations in low resolution tandem MS (MS2 $\mathrm{MS}^{3}, \mathrm{MS}^{4} \ldots$...) QqQ is mainly employed for targeted and quantitative analysis due to the high sensitivity obtained operating in Multiple Reaction Monitoring (MRM) mode. QTOF and Orbitrap analysers provide crucial structural information on the basis of the exact mass both molecular ions and fragments obtained by tandem-MS in high-resolution. Table 1 summarizes some representative applications for these MS analysers.

Carotenoid extracts from bacteria very often contain high concentrations of lipids (mainly triglycerides, TAGs) that are part of the cell membranes. The two main disadvantages of TAGs are high background noise in mass spectrometry with $\mathrm{APCI}(+)$ source and similar fragmentation patterns to carotenoid esters [7,67]. Thus, a clean-up step 
of the extracts is often required, that may involve biological methods using enzymes or physical methods using low temperatures. Although extraction and cleaning processes may seem time-consuming and cumbersome, especially the latter, they are suitable for subsequent MS identification [7,67,117].

\section{Applications in Food and Textile Industries}

Commercial carotenoids are mainly produced by synthesis. However, consumers' interest in products with carotenoids of natural origin has increased in recent years [32]. These compounds exhibit a wide range of applications in pharmaceutical, cosmetic, textile and food industries, among others [140,141]. In addition, the food industry has focused on manufacturing products that have striking colours of natural origin. Bacterial carotenoids applications in the food industry include the use of Brevibacterium linens in the fermentation of Limburger and Port-du-Salut cheeses, that produces the characteristic colour in these dairy products [96]. Likewise, a carotenoid that has shown wide versatility is astaxanthin. When this carotenoid is obtained from Paracoccus carotinifaciens, it which is used in the pigmentation of salmon and rainbow trout [142,143]; whereas astaxanthin from Mycobacterium lacticola is employed in fish feed for its antioxidant and photoprotective nature [144]. Additionally, xanthophylls such as canthaxanthin are profiteers in the pigmentation of processed foods such as margarine, butter, and confectionery. For example, canthaxanthin from Haloferax alexandrines increases the pigmentation of salmon flesh [145]. Also, zeaxanthin produced from Flavobacterium sp. is being used as an additive in poultry feed to change the colour of egg yolks and chicken skin to a darker yellow $[146,147]$. The industrial production of these carotenoids from bacteria such as Dietzia sp., Bradyrhizobium sp. or Gordonia alkanivorans could also potentially be employed in the food industry $[105,108,148]$.

On the other hand, the dyes used in the textile industry are largely of synthetic origin, involving highly polluting production processes. Thus, textile dyes extracted from bacteria can be a solution to this environmental problem. Serratia marcescens and Janthinobacterium lividum produce a pigment called violacein that has been shown to be an effective dye for different types of fabrics such as cotton, polyester, wool, and silk $[149,150]$. The desired colour intensity will depend on the time of exposure of the fabric to the bacteria and the amount of pigment that is extracted from them. Similarly, a yellow pigment from Thermomyces has been successfully used in the dyeing of silk [151,152]. Talaromyces verruculosus produces a red pigment that is capable to dye cotton [152]. In addition, Vibrio spp. Is a Gram-negative bacterium that has a bright red pigment, known as prodigiosin, which has been used to dye wool, nylon, and silk [32].

\section{Conclusions and Perspectives}

The great interest in the consumption of carotenoids of natural origin has encouraged the search of new sources for these compounds. Bacteria stand out as an excellent alternative due to the large variety of carotenoids that can be produced by different species. Moreover, bacteria can be considered a renewable source of carotenoids. In this paper, different methodologies frequently used for the extraction of bacterial carotenoids have been reviewed. Many of these methods involve multiple steps for extraction, concentration and resuspension of extracts, using large amounts of organic solvents such as hexane, $\mathrm{MeOH}$, and $\mathrm{CHCl}_{3}$, among others. Therefore, green extraction methodologies become environmentally friendly alternatives that should be considered in future research. Their use has been shown to be efficient in other microorganisms such as algae and fungi $[126,127,153]$. Moreover, bacterial carotenoids, very often found at low concentrations levels, makes the identification a challenging task. Therefore, the use of sensitive and high-throughput analytical methodologies such us HPLC-MS for qualitative and quantitative analysis of bacterial carotenoids becomes an essential requirement. The chromatographic separations have been developed with different stationary phases, 
although C18 and C30 columns are the most commonly used. The APCI source shows the best performance in the ionization of carotenoids, regardless of the mass analyser used. Thus, HPLC-DAD-APCI-MS/MS is the technique of choice for identifying carotenoids produced by new bacterial strains, that has been reported over the last few years [154163]. We predict a continuous development in the near future towards more sensitive and higher resolution methodologies that will allow to widen the coverage of carotenoids that can be identified in bacteria through the improvement of chromatographic columns, comprehensive methodologies in two-dimensional Liquid Chromatography (LCxLC), ionization processes and MS analysers. Finally, the search of new (or modified) bacteria as natural sources for carotenoids will remain a hot area of research for years to come.

Author Contributions: Conceptualization, C.L., C.C., and G.D.L.; methodology, G.D.L. G.A.R., E. I. and A.C.; validation, G.D.L. G.A.R., E. I. and A.C.; investigation, G.D.L.; writing-original draft preparation, G.D.L.; writing - review and editing, G.D.L., G.A.R., C.C., C.L. and A.C.; supervision, C.C., C.L., E. I. and A.C. All authors have read and agreed to the published version of the manuscript.

Funding: This research received no external funding.

Institutional Review Board Statement: Not applicable.

Informed Consent Statement: Not applicable.

Data Availability Statement: Not applicable.

Acknowledgments: The authors wish to thank to Colombian Ministerio de Ciencia, Tecnología e Innovación (MinCiencias) for PhD Fellowship to Gerson-Dirceu López (No. 785, Call 2017), as well as the support to No. 80740-532-2019 project and the Grant No. 120480763040. To Faculty of Sciences, Universidad de los Andes for research funds (INV-2018-48-1338, INV-2019-84-1824, INV-2019-20861843, and INV-2020-105-2084). Also, Ministerio de Ciencia y Universidades from Spain, funded the AGL2017-89417-R project and to Thomas Mccormack of Languages and Culture Department, Universidad de los Andes.

Conflicts of Interest: The authors declare no conflict of interest.

\section{References}

1. Liang, M.H.; Zhu, J.; Jiang, J.G. Carotenoids biosynthesis and cleavage related genes from bacteria to plants. Crit. Rev. Food Sci. Nutr. 2018, 58, 2314-2333, doi:10.1080/10408398.2017.1322552.

2. Amorim-Carrilho, K.T.; Cepeda, A.; Fente, C.; Regal, P. Review of methods for analysis of carotenoids. TrAC - Trends Anal. Chem. 2014, 56, 49-73, doi:10.1016/j.trac.2013.12.011.

3. Schaefer, B. Chapter 7 - Vitamins. In Natural Products in the Chemical Industry; Springer Berlin Heidelberg: Berlin, Heidelberg, 2014; pp. 589-640 ISBN 978-3-642-54460-6.

4. Valduga, E.; Oliveira Tatsch, P.; Tiggemann, L.; Treichel, H.; Toniazzo, G.; Zeni, J.; Luccio, M. Di; Fúrigo Júnior, A. Produção de carotenoides: microrganismos como fonte de pigmentos naturais. Quim. Nov. 2009, 32, 2429-2436.

5. Martínez, A.; Zeeshan, M.; Zaidi, A.; Sliwka, H.-R.; Razi Naqvi, K.; Partali, V. On infinitenes - Reliable calculation of $\lambda \infty$ and molecular modeling of lemniscate structured carotenoids. Comput. Theor. Chem. 2018, 1125, 133-141, doi:10.1016/j.comptc.2017.12.006.

6. Das, A.; Yoon, S.H.; Lee, S.H.; Kim, J.Y.; Oh, D.K.; Kim, S.W. An update on microbial carotenoid production: Application of recent metabolic engineering tools. Appl. Microbiol. Biotechnol. 2007, 77, 505-512, doi:10.1007/s00253-007-1206-3.

7. Mariutti, L.R.B.; Mercadante, A.Z. Carotenoid esters analysis and occurrence: What do we know so far? Arch. Biochem. Biophys. 2018, 648, 36-43, doi:10.1016/j.abb.2018.04.005.

8. Sliwka, H.R.; Partali, V. Key to xenobiotic carotenoids. Molecules 2012, 17, 2877-2928, doi:10.3390/molecules17032877.

9. Yabuzaki, J. Carotenoids Database: Structures, chemical fingerprints and distribution among organisms. Database 2017, 2017, bax004, doi:10.1093/database/bax004. 
10. Xue, L.; Chen, Y.Y.; Yan, Z.; Lu, W.; Wan, D.; Zhu, H. Staphyloxanthin: A potential target for antivirulence therapy. Infect. Drug Resist. 2019, 12, 2151-2160, doi:10.2147/IDR.S193649.

11. Kim, J.W.; Choi, B.H.; Kim, J.H.; Kang, H.J.; Ryu, H.; Lee, P.C. Complete genome sequence of Planococcus faecalis AJ003T,Planococcus faecalis AJ003 the type species of the genus Planococcus and a microbial C30 carotenoid producer. J. Biotechnol. 2018, 266, 72-76, doi:10.1016/j.jbiotec.2017.12.005.

12. Perez-Fons, L.; Steiger, S.; Khaneja, R.; Bramley, P.M.; Cutting, S.M.; Sandmann, G.; Fraser, P.D. Identification and the developmental formation of carotenoid pigments in the yellow/orange Bacillus spore-formers. Biochim. Biophys. Acta - Mol. Cell Biol. Lipids 2011, 1811, 177-185, doi:10.1016/j.bbalip.2010.12.009.

13. Asker, D.; Awad, T.S.; Beppu, T.; Ueda, K. Screening and profiling of natural ketocarotenoids from environmental aquatic bacterial isolates. Food Chem. 2018, 253, 247-254, doi:10.1016/j.foodchem.2018.01.066.

14. Kallscheuer, N.; Moreira, C.; Airs, R.; Llewellyn, C.A.; Wiegand, S.; Jogler, C.; Lage, O.M. Pink- and orange-pigmented Planctomycetes produce saproxanthin-type carotenoids including a rare C45 carotenoid. Environ. Microbiol. Rep. 2019, 11, 741-748, doi:10.1111/1758-2229.12796.

15. Heider, S.A.E.; Peters-Wendisch, P.; Wendisch, V.F.; Beekwilder, J.; Brautaset, T. Metabolic engineering for the microbial production of carotenoids and related products with a focus on the rare C50 carotenoids. Appl. Microbiol. Biotechnol. 2014, 98, 4355-4368, doi:10.1007/s00253-014-5693-8.

16. Saini, R.K.; Keum, Y.S. Carotenoid extraction methods: A review of recent developments. Food Chem. 2018, 240, 90-103, doi:10.1016/j.foodchem.2017.07.099.

17. Silva, T.R.; Tavares, R.S.N.; Canela-Garayoa, R.; Eras, J.; Rodrigues, M.V.N.; Neri-Numa, I.A.; Pastore, G.M.; Rosa, L.H.; Schultz, J.A.A.; Debonsi, H.M.; et al. Chemical Characterization and Biotechnological Applicability of Pigments Isolated from Antarctic Bacteria. Mar. Biotechnol. 2019, 21, 416-429, doi:10.1007/s10126-019-09892-z.

18. Giuffrida, D.; Sutthiwong, N.; Dugo, P.; Donato, P.; Cacciola, F.; Girard-Valenciennes, E.; Le Mao, Y.; Monnet, C.; Fouillaud, M.; Caro, Y.; et al. Characterisation of the C50 carotenoids produced by strains of the cheese-ripening bacterium Arthrobacter arilaitensis. Int. Dairy J. 2016, 55, 10-16, doi:10.1016/j.idairyj.2015.11.005.

19. Britton, G. Carotenoid research: History and new perspectives for chemistry in biological systems. Biochim. Biophys. Acta Mol. Cell Biol. Lipids 2020, 1865, 158699, doi:10.1016/j.bbalip.2020.158699.

20. Amengual, J. Bioactive properties of carotenoids in human health. Nutrients 2019, 11, 2388, doi:10.3390/nu11102388.

21. Buscemi, S.; Corleo, D.; Di Pace, F.; Petroni, M.L.; Satriano, A.; Marchesini, G. The effect of lutein on eye and extra-eye health. Nutrients 2018, 10, 1321, doi:10.3390/nu10091321.

22. Davinelli, S.; Nielsen, M.E.; Scapagnini, G. Astaxanthin in skin health, repair, and disease: A comprehensive review. Nutrients 2018, 10, 522, doi:10.3390/nu10040522.

23. Meléndez-Martínez, A.J.; Stinco, C.M.; Mapelli-Brahm, P. Skin carotenoids in public health and nutricosmetics: The emerging roles and applications of the UV radiation-absorbing colourless carotenoids phytoene and phytofluene. Nutrients 2019, 11, 1093, doi:10.3390/nu11051093.

24. Solymosi, K.; Latruffe, N.; Schoefs, B. Food colour additives of natural origin. In Colour Additives for Foods and Beverages; Elsevier Ltd., 2015; pp. 3-34 ISBN 9781782420118.

25. Asker, D. Isolation and Characterization of a Novel, Highly Selective Astaxanthin-Producing Marine Bacterium. J. Agric. Food Chem. 2017, 65, 9101-9109, doi:10.1021/acs.jafc.7b03556.

26. Ambati, R.R.; Gogisetty, D.; Aswathanarayana, R.G.; Ravi, S.; Bikkina, P.N.; Bo, L.; Yuepeng, S. Industrial potential of carotenoid pigments from microalgae: Current trends and future prospects. Crit. Rev. Food Sci. Nutr. 2019, 59, 1880-1902, doi:10.1080/10408398.2018.1432561.

27. Oslan, S.N.H.; Shoparwe, N.F.; Yusoff, A.H.; Rahim, A.A.; Chang, C.S.; Tan, J.S.; Oslan, S.N.; Arumugam, K.; Ariff, A. Bin; 
Sulaiman, A.Z.; et al. A review on haematococcus pluvialis bioprocess optimization of green and red stage culture conditions for the production of natural astaxanthin. Biomolecules 2021, 11, 256, doi:10.3390/biom11020256.

28. Zheng, Y.G.; Hu, Z.C.; Wang, Z.; Shen, Y.C. Large-scale production of astaxanthin by Xanthophyllomyces dendrorhous. Food Bioprod. Process. 2006, 84, 164-166, doi:10.1205/fbp.05030.

29. Sajjad, W.; Din, G.; Rafiq, M.; Iqbal, A.; Khan, S.; Zada, S.; Ali, B. Pigment production by cold - adapted bacteria and fungi : colorful tale of cryosphere with wide range applications. Extremophiles 2020, 24, 447-473, doi:10.1007/s00792-020-01180-2.

30. Kharangate-Lad, A.; Bhosle, S. Studies on Siderophore and Pigment Produced by an Adhered Bacterial Strain Halobacillus trueperi MXM-16 from the Mangrove Ecosystem of Goa, India. Indian J. Microbiol. 2016, 56, 461-466, doi:10.1007/s12088-0160591-7.

31. Sasidharan, P.; Raja, R.; Karthik, C.; Sharma, R.; P, I.A. Isolation and characterization of yellow pigment producing Exiguobacterium sps. J. Biochem. Technol. 2013, 4, 632-635.

32. Numan, M.; Bashir, S.; Mumtaz, R.; Tayyab, S.; Ur, N. Therapeutic applications of bacterial pigments : a review of current status and future opportunities. 3 Biotech 2018, 8, 1-15, doi:10.1007/s13205-018-1227-x.

33. Mussagy, C.U.; Winterburn, J.; Santos-Ebinuma, V.C.; Pereira, J.F.B. Production and extraction of carotenoids produced by microorganisms. Appl. Microbiol. Biotechnol. 2019, 103, 1095-1114, doi:10.1007/s00253-018-9557-5.

34. Mendes-Silva, T. de C.D.; Andrade, R.F. da S.; Ootani, M.A.; Mendes, P.V.D.; Sá, R.A. de Q.C. de; Silva, M.R.F. da; Souza, K.S.; Correia, M.T. dos S.; Silva, M.V. da; Oliveira, M.B.M. de Biotechnological Potential of Carotenoids Produced by Extremophilic Microorganisms and Application Prospects for the Cosmetics Industry. Adv. Microbiol. 2020, 10, 397-410, doi:10.4236/aim.2020.108029.

35. Narsing Rao, M.P.; Xiao, M.; Li, W.J. Fungal and bacterial pigments: Secondary metabolites with wide applications. Front. Microbiol. 2017, 8, 1113, doi:10.3389/fmicb.2017.01113.

36. Rapoport, A.; Guzhova, I.; Bernetti, L.; Buzzini, P.; Kieliszek, M.; Kot, A.M. Carotenoids and some other pigments from fungi and yeasts. Metabolites 2021, 11, 92, doi:10.3390/metabo11020092.

37. Rana, B.; Bhattacharyya, M.; Patni, B.; Arya, M.; Joshi, G.K. The Realm of Microbial Pigments in the Food Color Market. Front. Sustain. Food Syst. 2021, 5, 603892, doi:10.3389/fsufs.2021.603892.

38. Ram, S.; Mitra, M.; Shah, F.; Tirkey, S.R.; Mishra, S. Bacteria as an alternate biofactory for carotenoid production: A review of its applications, opportunities and challenges. J. Funct. Foods 2020, 67, 103867, doi:10.1016/j.jff.2020.103867.

39. Jinendiran, S.; Boopathi, S.; Sivakumar, N.; Selvakumar, G. Functional Characterization of Probiotic Potential of Novel Pigmented Bacterial Strains for Aquaculture Applications. Probiotics Antimicrob. Proteins 2019, 11, 186-197, doi:10.1007/s12602-017-9353-z.

40. Venil, C.K.; Dufossé, L.; Renuka Devi, P. Bacterial Pigments: Sustainable Compounds With Market Potential for Pharma and Food Industry. Front. Sustain. Food Syst. 2020, 4, 1-17, doi:10.3389/fsufs.2020.00100.

41. Velmurugan, P.; Venil, C.K.; Veera Ravi, A.; Dufossé, L. Marine Bacteria Is the Cell Factory to Produce Bioactive Pigments: A Prospective Pigment Source in the Ocean. Front. Sustain. Food Syst. 2020, 4, 589655, doi:10.3389/fsufs.2020.589655.

42. Pailliè-Jiménez, M.E.; Stincone, P.; Brandelli, A. Natural Pigments of Microbial Origin. Front. Sustain. Food Syst. 2020, 4, 1-8, doi:10.3389/fsufs.2020.590439.

43. Mussagy, C.U.; Khan, S.; Kot, A.M. Current developments on the application of microbial carotenoids as an alternative to synthetic pigments. Crit. Rev. Food Sci. Nutr. 2021, 1-15, doi:10.1080/10408398.2021.1908222.

44. Zhu, Y.; Graham, J.E.; Ludwig, M.; Xiong, W.; Alvey, R.M.; Shen, G.; Bryant, D.A. Roles of xanthophyll carotenoids in protection against photoinhibition and oxidative stress in the cyanobacterium Synechococcus sp. strain PCC 7002. Arch. Biochem. Biophys. 2010, 504, 86-99, doi:10.1016/j.abb.2010.07.007.

45. Maoka, T. Carotenoids as natural functional pigments. J. Nat. Med. 2020, 74, 1-16, doi:10.1007/s11418-019-01364-x. 
46. Frengova, G.I.; Beshkova, D.M. Carotenoids from Rhodotorula and Phaffia: Yeasts of biotechnological importance. J. Ind. Microbiol. Biotechnol. 2009, 36, 163-180, doi:10.1007/s10295-008-0492-9.

47. Córdova, P.; Baeza, M.; Cifuentes, V.; Alcaíno, J. Microbiological Synthesis of Carotenoids: Pathways and Regulation. In Progress in Carotenoid Research pigments; Queiroz Zepka, L., Jacob-Lopes, E., Rosso, V.V., Eds.; IntechOpen, 2018; pp. 63-83 ISBN 9781626239777.

48. Niu, F.X.; Lu, Q.; Bu, Y.F.; Liu, J.Z. Metabolic engineering for the microbial production of isoprenoids: Carotenoids and isoprenoid-based biofuels. Synth. Syst. Biotechnol. 2017, 2, 167-175, doi:10.1016/j.synbio.2017.08.001.

49. Wang, C.; Zhao, S.; Shao, X.; Park, J. Bin; Jeong, S.H.; Park, H.J.; Kwak, W.J.; Wei, G.; Kim, S.W. Challenges and tackles in metabolic engineering for microbial production of carotenoids. Microb. Cell Fact. 2019, 18, 1-8, doi:10.1186/s12934-019-11051.

50. Zhang, C. Biosynthesis of Carotenoids and Apocarotenoids by Microorganisms and Their Industrial Potential. In Progress in Carotenoid Research pigments; Queiroz Zepka, L., Jacob-Lopes, E., Rosso, V.V., Eds.; 2018; pp. 85-105 ISBN 9781626239777.

51. Rowles, J.L.; Erdman, J.W. Carotenoids and their role in cancer prevention. Biochim. Biophys. Acta - Mol. Cell Biol. Lipids 2020, 1865, 158613, doi:10.1016/j.bbalip.2020.158613.

52. Perera, C.O.; Yen, G.M. Functional properties of carotenoids in human health. Int. J. Food Prop. 2007, 10, 201-230, doi:10.1080/10942910601045271.

53. Bohn, T.; Desmarchelier, C.; El, S.N.; Keijer, J.; Van Schothorst, E.; Rühl, R.; Borel, P. $\beta$-Carotene in the human body: Metabolic bioactivation pathways - From digestion to tissue distribution and excretion. Proc. Nutr. Soc. 2019, 78, 68-87, doi:10.1017/S0029665118002641.

54. Arunkumar, R.; Gorusupudi, A.; Bernstein, P.S. The macular carotenoids: A biochemical overview. Biochim. Biophys. Acta Mol. Cell Biol. Lipids 2020, 1865, 158617, doi:10.1016/j.bbalip.2020.158617.

55. Aziz, E.; Batool, R.; Akhtar, W.; Rehman, S.; Shahzad, T.; Malik, A.; Shariati, M.A.; Laishevtcev, A.; Plygun, S.; Heydari, M.; et al. Xanthophyll: Health benefits and therapeutic insights. Life Sci. 2020, 240, 117104, doi:10.1016/j.lfs.2019.117104.

56. Pérez-Gálvez, A.; Roca, M. Recent Developments in the Analysis of Carotenoids by Mass Spectrometry. In Progress in Carotenoid Research; IntechOpen, 2018; pp. 17-44.

57. Kumar, S.V.; Taylor, G.; Hasim, S.; Collier, C.P.; Farmer, A.T.; Campagna, S.R.; Bible, A.N.; Doktycz, M.J.; Morrell-Falvey, J. Loss of carotenoids from membranes of Pantoea sp. YR343 results in altered lipid composition and changes in membrane biophysical properties. Biochim. Biophys. Acta - Biomembr. 2019, 1861, 1338-1345, doi:10.1016/j.bbamem.2019.05.009.

58. Nemer, G.; Louka, N.; Vorobiev, E.; Salameh, D.; Nicaud, J.-M.; Maroun, R.G.; Koubaa, M. Mechanical Cell Disruption Technologies for the Extraction of Dyes and Pigments from Microorganisms: A Review. Fermentation 2021, 7, 36, doi:10.3390/fermentation7010036.

59. Larrosa, A.P.Q.; Camara, Á.S.; Moura, J.M.; Pinto, L.A.A. Spirulina sp. biomass dried/disrupted by different methods and their application in biofilms production. Food Sci. Biotechnol. 2018, 27, 1659-1665, doi:10.1007/s10068-018-0397-y.

60. Sowmya, R.; Sachindra, N.M. Carotenoid production by Formosa sp. KMW, a marine bacteria of Flavobacteriaceae family: Influence of culture conditions and nutrient composition. Biocatal. Agric. Biotechnol. 2015, 4, 559-567, doi:10.1016/j.bcab.2015.08.018.

61. Park, W.S.; Kim, H.J.; Li, M.; Lim, D.H.; Kim, J.; Kwak, S.S.; Kang, C.M.; Ferruzzi, M.G.; Ahn, M.J. Two classes of pigments, carotenoids and c-phycocyanin, in spirulina powder and their antioxidant activities. Molecules 2018, 23, 2065, doi:10.3390/molecules23082065.

62. Gu, Z.; Deming, C.; Yongbin, H.; Zhigang, C.; Feirong, G. Optimization of carotenoids extraction from Rhodobacter sphaeroides. LWT - Food Sci. Technol. 2008, 41, 1082-1088, doi:10.1016/j.lwt.2007.07.005.

63. Montero, O.; Macìs-Sánchez, M.D.; Lama, C.M.; Lubián, L.M.; Mantell, C.; Rodríguez, M.; De La Ossa, E.M. Supercritical 
$\mathrm{CO} 2$ extraction of $\beta$-carotene from a marine strain of the cyanobacterium Synechococcus species. J. Agric. Food Chem. 2005, 53, 9701-9707, doi:10.1021/jf051283n.

64. Ron, E.Y.C.; Plaza, M.; Kristjansdottir, T.; Sardari, R.R.R.; Bjornsdottir, S.H.; Gudmundsson, S.; Hreggvidsson, G.O.; Turner, C.; van Niel, E.W.J.; Nordberg-Karlsson, E. Characterization of carotenoids in Rhodothermus marinus. Microbiologyopen 2018, 7, 7:e536, doi:10.1002/mbo3.536.

65. Sowmya, R.; Sachindra, N.M. Biochemical and molecular characterization of carotenogenic flavobacterial isolates from marine waters. Polish J. Microbiol. 2016, 65, 77-88, doi:10.5604/17331331.1197278.

66. Henke, N.A.; Frohwitter, J.; Peters-Wendisch, P.; Wendisch, V.F. Carotenoid production by recombinant Corynebacterium glutamicum: Strain construction, cultivation, extraction, and quantification of carotenoids and terpenes. In Methods in Molecular Biology; Humana Press Inc., 2018; Vol. 1852, pp. 127-141.

67. Mercadante, A.Z.; Rodrigues, D.B.; Petry, F.C.; Mariutti, L.R.B. Carotenoid esters in foods - A review and practical directions on analysis and occurrence. Food Res. Int. 2017, 99, 830-850, doi:10.1016/j.foodres.2016.12.018.

68. Kim, S.H.; Lee, P.C. Functional expression and extension of staphylococcal staphyloxanthin biosynthetic pathway in Escherichia coli. J. Biol. Chem. 2012, 287, 21575-21583, doi:10.1074/jbc.M112.343020.

69. Cerón-García, M.C.; González-López, C. V.; Camacho-Rodríguez, J.; López-Rosales, L.; García-Camacho, F.; Molina-Grima, E. Maximizing carotenoid extraction from microalgae used as food additives and determined by liquid chromatography (HPLC). Food Chem. 2018, 257, 316-324, doi:10.1016/j.foodchem.2018.02.154.

70. Pelz, A.; Wieland, K.P.; Putzbach, K.; Hentschel, P.; Albert, K.; Götz, F. Structure and biosynthesis of staphyloxanthin from Staphylococcus aureus. J. Biol. Chem. 2005, 280, 32493-32498, doi:10.1074/jbc.M505070200.

71. Mishra, N.N.; Liu, G.Y.; Yeaman, M.R.; Nast, C.C.; Proctor, R.A.; McKinnell, J.; Bayer, A.S. Carotenoid-related alteration of cell membrane fluidity impacts Staphylococcus aureus susceptibility to host defense peptides. Antimicrob. Agents Chemother. 2011, 55, 526-531, doi:10.1128/AAC.00680-10.

72. Rezaeeyan, Z.; Safarpour, A.; Amoozegar, M.A.; Babavalian, H.; Tebyanian, H.; Shakeri, F. High carotenoid production by a halotolerant bacterium, Kocuria sp. Strain QWT-12 and anticancer activity of its carotenoid. EXCLI J. 2017, 16, 840-851, doi:10.17179/excli2017-218.

73. Hartz, P.; Milhim, M.; Trenkamp, S.; Bernhardt, R.; Hannemann, F. Characterization and engineering of a carotenoid biosynthesis operon from Bacillus megaterium. Metab. Eng. 2018, 49, 47-58, doi:10.1016/j.ymben.2018.07.017.

74. Jing, Y.; Liu, H.; Xu, W.; Yang, Q. Amelioration of the DSS-induced colitis in mice by pretreatment with 4,4'diaponeurosporene-producing Bacillus subtilis. Exp. Ther. Med. 2017, 14, 6069-6073, doi:10.3892/etm.2017.5282.

75. Ye, R.W.; Yao, H.; Stead, K.; Wang, T.; Tao, L.; Cheng, Q.; Sharpe, P.L.; Suh, W.; Nagel, E.; Arcilla, D.; et al. Construction of the astaxanthin biosynthetic pathway in a methanotrophic bacterium Methylomonas sp. strain 16a. J. Ind. Microbiol. Biotechnol. 2007, 34, 289-299, doi:10.1007/s10295-006-0197-x.

76. Iwata, S.; Imai, T.; Shimazawa, M.; Ishibashi, T.; Hayashi, M.; Hara, H.; Nakamura, S. Protective effects of the astaxanthin derivative, adonixanthin, on brain hemorrhagic injury. Brain Res. 2018, 1698, 130-138, doi:10.1016/j.brainres.2018.08.009.

Vila, E.; Hornero-Méndez, D.; Azziz, G.; Lareo, C.; Saravia, V. Carotenoids from heterotrophic bacteria isolated from Fildes Peninsula, King George Island, Antarctica. Biotechnol. Reports 2019, 21, e00306, doi:10.1016/j.btre.2019.e00306.

78. Buddhi, S.; Suresh, G.; Gupta, D.; Sasikala, C.; Ramana, C. V. Afifella aestuarii sp. nov., a phototrophic bacterium. Int. J. Syst. Evol. Microbiol. 2020, 70, 327-333, doi:10.1099/ijsem.0.003756.

79. Chen, Y.; Xie, B.; Yang, J.; Chen, J.; Sun, Z. Identification of microbial carotenoids and isoprenoid quinones from Rhodococcus sp. B7740 and its stability in the presence of iron in model gastric conditions. Food Chem. 2018, 240, 204-211, doi:10.1016/j.foodchem.2017.06.067.

80. Mijts, B.N.; Lee, P.C.; Schmidt-Dannert, C. Identification of a carotenoid oxygenase synthesizing acyclic xanthophylls: 
Combinatorial biosynthesis and directed evolution. Chem. Biol. 2005, 12, 453-460, doi:10.1016/j.chembiol.2005.02.010.

81. Kim, J.H.; Kang, H.J.; Yu, B.J.; Kim, S.C.; Lee, P.C. Planococcus faecalis sp. Nov., a carotenoidproducing species isolated from stools of antarctic penguins. Int. J. Syst. Evol. Microbiol. 2015, 65, 3373-3378, doi:10.1099/ijsem.0.000423.

82. Heo, J.; Kim, S.H.; Lee, P.C. New insight into the cleavage reaction of Nostoc sp. strain PCC 7120 carotenoid cleavage dioxygenase in natural and nonnatural carotenoids. Appl. Environ. Microbiol. 2013, 79, 3336-3345, doi:10.1128/AEM.00071-13.

83. Tao, L.; Cheng, Q. Novel $\beta$-carotene ketolases from non-photosynthetic bacteria for canthaxanthin synthesis. Mol. Genet. Genomics 2004, 272, 530-537, doi:10.1007/s00438-004-1083-8.

84. Tao, L.; Wagner, L.W.; Rouvière, P.E.; Cheng, Q. Metabolic engineering for synthesis of aryl carotenoids in Rhodococcus. Appl. Microbiol. Biotechnol. 2006, 70, 222-228, doi:10.1007/s00253-005-0064-0.

85. Ganapathy, A.; Jayavel, S.; Natesan, S. Draft Genome Sequence of Carotenoid Producing Yellow Pigmented Planococcus maritimus MKU009 . J. Genomics 2016, 4, 23-25, doi:10.7150/jgen.15533.

86. Shindo, K.; Endo, M.; Miyake, Y.; Wakasugi, K.; Morritt, D.; Bramley, P.M.; Fraser, P.D.; Kasai, H.; Misawa, N. Methyl Glucosyl-3,4-dehydro-apo-8'-lycopenoate, a Novel Antioxidative Glyco-C30-carotenoic Acid Produced by a Marine Bacterium Planococcus maritimus. J. Antibiot. 2008, 61, 729-735.

87. Hyeon, J.W.; Jeon, C.O. Roseomonas aerofrigidensis sp. nov., isolated from an air conditioner. Int. J. Syst. Evol. Microbiol. 2017, 67, 4039-4044, doi:10.1099/ijsem.0.002246.

88. Ramana, V.V.; Sasikala, C.; Ramaprasad, E.V.V.; Ramana, C.V. Description of Ectothiorhodospira salini sp. nov. J. Gen. Appl. Microbiol. 2010, 56, 313-319, doi:10.2323/jgam.56.313.

89. Widyastuti, Y.; Nugraheni, S.A.; Khoeri, M.M.; Kusmita, L.; Radjasa, O.K. Characterization of carotenoid pigments from bacterial symbionts of seagrass Thalassia hemprichii Knowledge Management on Low Emission Strategy into Coastal Management in NTB and NTB Province View project DNA Barcoding View project CHARACTERIZATION OF CAR. J. Coast. Dev. 2010, 14, 51-60, doi:10.13140/RG.2.1.3348.6562.

90. Arpin, N.; Liaaen-Jensen, S.; Trouilloud, M. Bacterial carotenoids. XXXVIII. C 50 -carotenoids 9. Isolation of decaprenoxanthin mono- and diglucoside from an Arthrobacter sp. Acta Chem. Scand. 1972, 26, 2524-2526, doi:10.3891/acta.chem.scand.26-2524.

91. Zhao, C.; Yue, H.; Cheng, Q.; Chen, S.; Yang, S. What caused the formation of the absorption maximum at $421 \mathrm{~nm}$ in vivo spectra of rhodopseudomonas palustris. Photochem. Photobiol. 2014, 90, 1287-1292, doi:10.1111/php.12334.

92. Ramaprasad, E.V.V.; Sasikala, C.; Ramana, C. V. Neurosporene is the major carotenoid accumulated by Rhodobacter viridis JA737. Biotechnol. Lett. 2013, 35, 1093-1097, doi:10.1007/s10529-013-1181-y.

93. Rahul, K.; Azmatunnisa, M.; Sasikala, C.H.; Ramana, C. V. Hoeflea olei sp. Nov., a diesel-oil-degrading, anoxygenic, phototrophic bacterium isolated from backwaters and emended description of the genus Hoeflea. Int. J. Syst. Evol. Microbiol. 2015, 65, 2403-2409, doi:10.1099/ijs.0.000277.

94. Takaichi, S.; Maoka, T.; Akimoto, N.; Carmona, M.L.; Yamaoka, Y. Carotenoids in a corynebacterineae, Gordonia terrae AIST1: Carotenoid glucosyl mycoloyl esters. Biosci. Biotechnol. Biochem. 2008, 72, 2615-2622, doi:10.1271/bbb.80299.

95. Jiménez, M.E.P.; Pinilla, C.M.B.; Rodrigues, E.; Brandelli, A. Extraction and partial characterisation of antioxidant pigment produced by Chryseobacterium sp. kr6. Nat. Prod. Res. 2019, 33, 1541-1549, doi:10.1080/14786419.2017.1423304.

96. Applique, L.D.M.; Alimentaires, I. Production of carotenoids by Brevibacterium linens : variation among strains , kinetic aspects and HPLC profiles. J. Ind. Microbiol. Biotechnol. 2000, 24, 64-70, doi:10.1038/sj.jim.2900761.

97. Kulichevskaya, I.S.; Guzev, V.S.; Gorlenko, V.M.; Liesack, W.; Dedysh, S.N. Rhodoblastus sphagnicola sp. nov., a novel acidophilic purple non-sulfur bacterium form Sphagnum peat bog. Int. J. Syst. Evol. Microbiol. 2006, 56, 1397-1402, doi:10.1099/ijs.0.63962-0.

98. Alvares, J.J.; Furtado, I.J. Characterization of multicomponent antioxidants from Haloferax alexandrinus GUSF-1 (KF796625). 
3 Biotech 2021, 11, 1-12, doi:10.1007/s13205-020-02584-9.

99. Asker, D. High throughput screening and profiling of high-value carotenoids from a wide diversity of bacteria in surface seawater. Food Chem. 2018, 261, 103-111, doi:10.1016/j.foodchem.2018.03.109.

100. Liu, H.; Tan, K.S.; Zhang, X.; Zhang, H.; Cheng, D.; Ting, Y.; Li, S.; Ma, H.; Zheng, H. Comparison of Gut Microbiota Between Golden and Brown Noble Scallop Chlamys nobilis and Its Association With Carotenoids. Front. Microbiol. 2020, 11, 36, doi:10.3389/fmicb.2020.00036.

101. Yokoyama, A.; Izumida, H.; Miki, W. Production of astaxanthin and 4-ketozeaxanthin by the marine bacterium, agrobacterium aurantiacum. Biosci. Biotechnol. Biochem. 1994, 58, 1842-1844, doi:10.1271/bbb.58.1842.

102. Liu, H.; Zhang, C.; Zhang, X.; Tan, K.; Zhang, H.; Cheng, D.; Ye, T.; Li, S.; Ma, H.; Zheng, H. A novel carotenoids-producing marine bacterium from noble scallop Chlamys nobilis and antioxidant activities of its carotenoid compositions. Food Chem. 2020, 320, 126629, doi:10.1016/j.foodchem.2020.126629.

103. Asker, D.; Beppu, T.; Ueda, K. Sphingomonas astaxanthinifaciens sp. nov., a novel astaxanthin-producing bacterium of the family Sphingomonadaceae isolated from Misasa, Tottori, Japan. FEMS Microbiol. Lett. 2007, 273, 140-148, doi:10.1111/j.15746968.2007.00760.x.

104. Mageswari, A.; Subramanian, P.; Srinivasan, R.; Karthikeyan, S.; Gothandam, K.M. Astaxanthin from psychrotrophic Sphingomonas faeni exhibits antagonism against food-spoilage bacteria at low temperatures. Microbiol. Res. 2015, 179, 38-44, doi:10.1016/j.micres.2015.06.010.

105. Silva, T.P.; Paixão, S.M.; Alves, L. Ability of: Gordonia alkanivorans strain 1B for high added value carotenoids production. RSC Adv. 2016, 6, 58055-58063, doi:10.1039/c6ra08126f.

106. Ye, R.W.; Stead, K.J.; Yao, H.; He, H. Mutational and functional analysis of the $\beta$-carotene ketolase involved in the production of canthaxanthin and astaxanthin. Appl. Environ. Microbiol. 2006, 72, 5829-5837, doi:10.1128/AEM.00918-06.

107. Lorquin, J.; Molouba, F.; Dreyfus, B.L. Identification of the Carotenoid Pigment Canthaxanthin from Photosynthetic Bradyrhizobium Strains. Appl. Environ. Microbiol. 1997, 63, 1151-1154.

108. Venugopalan, V.; Tripathi, S.K.; Nahar, P.; Saradhi, P.P.; Das, R.H.; Gautam, H.K. Characterization of canthaxanthin isomers isolated from a new soil Dietzia sp. and their antioxidant activities. J. Microbiol. Biotechnol. 2013, 23, 237-245, doi:10.4014/jmb.1203.03032.

109. Graham, J.E.; Lecomte, J.T.J.; Bryant, D.A. Synechoxanthin, an aromatic C40 xanthophyll that is a major carotenoid in the cyanobacterium Synechococcus sp. PCC 7002. J. Nat. Prod. 2008, 71, 1647-1650, doi:10.1021/np800310b.

110. Asker, D.; Beppu, T.; Ueda, K. Mesoflavibacter zeaxanthinifaciens gen. nov., sp. nov., a novel zeaxanthin-producing marine bacterium of the family Flavobacteriaceae. Syst. Appl. Microbiol. 2007, 30, 291-296, doi:10.1016/j.syapm.2006.12.003.

111. Sarnaik, A.; Nambissan, V.; Pandit, R.; Lali, A. Recombinant Synechococcus elongatus PCC 7942 for improved zeaxanthin production under natural light conditions. Algal Res. 2018, 36, 139-151, doi:10.1016/j.algal.2018.10.021.

112. Berry, A.; Janssens, D.; Hümbelin, M.; Jore, J.P.M.; Hoste, B.; Cleenwerck, I.; Vancanneyt, M.; Bretzel, W.; Mayer, A.F.; LopezUlibarri, R.; et al. Paracoccus zeaxanthinifaciens sp. nov., a zeaxanthin-producing bacterium. Int. J. Syst. Evol. Microbiol. 2003, 53, 231-238, doi:10.1099/ijs.0.02368-0.

113. Antolak, H.; Oracz, J.; Otlewska, A.; Zyzelewicz, D.; Kregiel, D. Identification of Carotenoids and Isoprenoid Quinones from Asaia lannensis and Asaia bogorensis. Molecules 2017, 22, 1608, doi:10.3390/molecules22101608.

114. Jinendiran, S.; Dahms, H.U.; Dileep Kumar, B.S.; Kumar Ponnusamy, V.; Sivakumar, N. Diapolycopenedioic-acid-diglucosyl ester and keto-myxocoxanthin glucoside ester: Novel carotenoids derived from Exiguobacterium acetylicum S01 and evaluation of their anticancer and anti-inflammatory activities. Bioorg. Chem. 2020, 103, 104149, doi:10.1016/j.bioorg.2020.104149.

115. Jinendiran, S.; Dileep Kumar, B.S.; Dahms, H.U.; Arulanandam, C.D.; Sivakumar, N. Optimization of submerged 
fermentation process for improved production of $\beta$-carotene by Exiguobacterium acetylicum S01. Heliyon 2019, 5, e01730, doi:10.1016/j.heliyon.2019.e01730.

116. Fukaya, Y.; Takemura, M.; Koyanagi, T.; Maoka, T.; Shindo, K.; Misawa, N. Structural and functional analysis of the carotenoid biosynthesis genes of a Pseudomonas strain isolated from the excrement of Autumn Darter. Biosci. Biotechnol. Biochem. 2018, 82, 1043-1052, doi:10.1080/09168451.2017.1398069.

117. López, G.-D.; Suesca, E.; Álvarez-Rivera, G.; Rosato, A.E.; Ibáñez, E.; Cifuentes, A.; Leidy, C.; Carazzone, C. Carotenogenesis of Staphylococcus aureus: New insights and impact on membrane biophysical properties. Biochim. Biophys. Acta - Mol. Cell Biol. Lipids 2021, 1866, 158941, doi:10.1016/j.bbalip.2021.158941.

118. Saha, S.; Walia, S.; Sharma, K.; Banerjee, K. Suitability of stationary phase for LC analysis of biomolecules. Crit. Rev. Food Sci. Nutr. 2019, doi:10.1080/10408398.2019.1665494.

119. Turcsi, E.; Nagy, V.; Deli, J. Study on the elution order of carotenoids on endcapped C18 and C30 reverse silica stationary phases. A review of the database. J. Food Compos. Anal. 2016, 47, 101-112, doi:10.1016/j.jfca.2016.01.005.

120. Jin, H.; Lao, Y.M.; Zhou, J.; Zhang, H.J.; Cai, Z.H. Simultaneous determination of 13 carotenoids by a simple C18 columnbased ultra-high-pressure liquid chromatography method for carotenoid profiling in the astaxanthin-accumulating Haematococcus pluvialis. J. Chromatogr. A 2017, 1488, 93-103, doi:10.1016/j.chroma.2017.01.088.

121. Kaiser, P.; Geyer, R.; Surmann, P.; Fuhrmann, H. LC-MS method for screening unknown microbial carotenoids and isoprenoid quinones. J. Microbiol. Methods 2012, 88, 28-34, doi:10.1016/j.mimet.2011.10.001.

122. Bridoux, M.C.; Sobiechowska, M.; Pérez-Fuentetaja, A.; Alben, K.T. LC-PDA/APClitMS identification of algal carotenoid and oxysterol precursors to fatty acid esters in hydrolyzed extracts of the freshwater mussel Dreissena bugensis. Anal. Bioanal. Chem. 2017, 409, 6745-6760, doi:10.1007/s00216-017-0562-9.

123. Abate-Pella, D.; Freund, D.M.; Slovin, J.P.; Hegeman, A.D.; Cohen, J.D. An improved method for fast and selective separation of carotenoids by LC-MS. J. Chromatogr. B Anal. Technol. Biomed. Life Sci. 2017, 1067, 34-37, doi:10.1016/j.jchromb.2017.09.039.

124. Hrvolová, B.; Martínez-Huélamo, M.; Colmán-Martínez, M.; Hurtado-Barroso, S.; Lamuela-Raventós, R.M.; Kalina, J. Development of an advanced HPLC-MS/MS method for the determination of carotenoids and fat-soluble vitamins in human plasma. Int. J. Mol. Sci. 2016, 17, 1719, doi:10.3390/ijms17101719.

125. Schex, R.; Lieb, V.M.; Jiménez, V.M.; Esquivel, P.; Schweiggert, R.M.; Carle, R.; Steingass, C.B. HPLC-DAD-APCI/ESI-MSn analysis of carotenoids and $\alpha$-tocopherol in Costa Rican Acrocomia aculeata fruits of varying maturity stages. Food Res. Int. 2018, 105, 645-653, doi:10.1016/j.foodres.2017.11.041.

126. Gallego, R.; Martínez, M.; Cifuentes, A.; Ibáñez, E.; Herrero, M. Development of a Green Downstream Process for the Valorization of Porphyridium cruentum Biomass. Molecules 2019, 24, 1564, doi:10.3390/molecules24081564.

127. Gallego, R.; Arena, K.; Dugo, P.; Mondello, L.; Ibáñez, E.; Herrero, M. Application of compressed fluid - based extraction and purification procedures to obtain astaxanthin-enriched extracts from Haematococcus pluvialis and characterization by comprehensive two-dimensional liquid chromatography coupled to mass spectrometry. Anal. Bioanal. Chem. 2020, 412, 589599, doi:10.1007/s00216-019-02287-y.

128. Castro-Puyana, M.; Pérez-Sánchez, A.; Valdés, A.; Ibrahim, O.H.M.; Suarez-Álvarez, S.; Ferragut, J.A.; Micol, V.; Cifuentes, A.; Ibáñez, E.; García-Cañas, V. Pressurized liquid extraction of Neochloris oleoabundans for the recovery of bioactive carotenoids with anti-proliferative activity against human colon cancer cells. Food Res. Int. 2017, 99, 1048-1055, doi:10.1016/j.foodres.2016.05.021.

129. van Breemen, R.B. Mass Spectrometry of Carotenoids. In Current Protocols in Food Analytical Chemistry; 2001; p. F2.4.1-F2.4.13.

130. Carnevale, J.; Cole, E.R.; Nelson, D.; Shannon, J.S. Chemical Ionization Mass Spectrometry of Carotenoids. Biomed. Mass Spectrom. 1978, 5, 641-546, doi:10.1002/bms.1200051109.

131. Breemen, R.B. Van; Schmitz, H.H.; Schwartz, S.J. Fast Atom Bombardment Tandem Mass Spectrometry of Carotenoids. J. 
Agric. Food Chem 1995, 43, 384-389, doi:10.1021/jf00050a024.

132. Van Breemen, R.B.; Dong, L.; Pajkovic, N.D. Atmospheric pressure chemical ionization tandem mass spectrometry of carotenoids. Int. J. Mass Spectrom. 2012, 312, 163-172, doi:10.1016/j.ijms.2011.07.030.

133. Huang, S.; Xu, J.; Wu, J.; Hong, H.; Chen, G.; Jiang, R.; Zhu, F.; Liu, Y.; Ouyang, G. Rapid detection of five anesthetics in tilapias by in vivo solid phase microextraction coupling with gas chromatography-mass spectrometry. Talanta 2017, 168, 263268, doi:10.1016/j.talanta.2017.03.045.

134. Schöner, T.A.; Gassel, S.; Osawa, A.; Tobias, N.J.; Okuno, Y.; Sakakibara, Y.; Shindo, K.; Sandmann, G.; Bode, H.B. Aryl Polyenes, a Highly Abundant Class of Bacterial Natural Products, Are Functionally Related to Antioxidative Carotenoids. ChemBioChem 2016, 17, 247-253, doi:10.1002/cbic.201500474.

135. Stafsnes, M.H.; Dybwad, M.; Brunsvik, A.; Bruheim, P. Large scale MALDI-TOF MS based taxa identification to identify novel pigment producers in a marine bacterial culture collection. Antonie Van Leeuwenhoek 2013, 103, 603-615, doi:10.1007/s10482-012-9844-6.

136. Yoshida, K.; Ueda, S.; Maeda, I. Carotenoid production in Bacillus subtilis achieved by metabolic engineering. Biotechnol. Lett. 2009, 31, 1789-1793, doi:10.1007/s10529-009-0082-6.

137. Manikandan, M.; Hasan, N.; Wu, H.F. Rapid detection of haloarchaeal carotenoids via liquid-liquid microextraction enabled direct TLC MALDI-MS. Talanta 2013, 107, 167-175, doi:10.1016/j.talanta.2013.01.005.

138. Neto, F.C.; Guaratini, T.; Costa-Lotufo, L.; Colepicolo, P.; Gates, P.J.; Lopes, N.P. Re-investigation of the fragmentation of protonated carotenoids by electrospray ionization and nanospray tandem mass spectrometry. Rapid Commun. Mass Spectrom. 2016, 30, 1540-1548, doi:10.1002/rcm.7589.

139. Rivera, S.; Vilaró, F.; Canela, R. Determination of carotenoids by liquid chromatography/mass spectrometry: Effect of several dopants. Anal. Bioanal. Chem. 2011, 400, 1339-1346, doi:10.1007/s00216-011-4825-6.

140. Zerres, S.; Stahl, W. Carotenoids in human skin. Biochim. Biophys. Acta - Mol. Cell Biol. Lipids 2020, 1865, 158588, doi:10.1016/j.bbalip.2019.158588.

141. Clugston, R.D. Carotenoids and fatty liver disease: Current knowledge and research gaps. Biochim. Biophys. Acta - Mol. Cell Biol. Lipids 2020, 1865, 158597, doi:10.1016/j.bbalip.2019.158597.

142. Grewe, C.; Menge, S.; Griehl, C. Enantioselective separation of all-E-astaxanthin and its determination in microbial sources. J. Chromatogr. A 2007, 1166, 97-100, doi:10.1016/j.chroma.2007.08.002.

143. Bories, G.; Brantom, P.; Barberà, J.B. De; Chesson, A.; Sandro, P.; Debski, B.; Dierick, N.; Franklin, A.; Gropp, J.; Halle, I.; et al. Opinion of the Scientific Panel on additives and products or substances used in animal feed (FEEDAP) on Safety and efficacy of Panaferd-AX (red carotenoid-rich bacterium Paracoccus carotinifaciens) as feed additive for salmon and trout. EFSA J. 2007, 5, 1-30, doi:10.2903/j.efsa.2007.546.

144. Kirti, K.; Amita, S.; Priti, S.; Mukesh Kumar, A.; Jyoti, S. Colorful World of Microbes: Carotenoids and Their Applications. Adv. Biol. 2014, 2014, 1-13, doi:10.1155/2014/837891.

145. Manikandan, K.; Felix, N.; Prabu, E. A review on the application and effect of carotenoids with respect to canthaxanthin in the culture of fishes and crustaceans. Int. J. Fish. Aquat. Stud. 2020, 8, 128-133, doi:10.22271/fish.2020.v8.i5b.2314.

146. Pasarin, D.; Rovinaru, C. Sources of Carotenoids and Their Uses As Animal Feed Additives - a Review. Sci. Pap. D-Animal Sci. 2018, 61, 74-85.

147. Masetto, A.; Flores-Cotera, L.B.; Díaz, C.; Langley, E.; Sanchez, S. Application of a complete factorial design for the production of zeaxanthin by Flavobacterium sp. J. Biosci. Bioeng. 2001, 92, 55-58, doi:10.1263/jbb.92.55.

148. Barredo, J.-L. Microbial Carotenoids; Barreiro, C., Barredo, J.-L., Eds.; Methods in Molecular Biology; Springer New York: New York, NY, 2018; Vol. 1852; ISBN 978-1-4939-8741-2.

149. Chatragadda, R.; Dufossé, L. Ecological and biotechnological aspects of pigmented microbes: A way forward in development 
of food and pharmaceutical grade pigments. Microorganisms 2021, 9, 1-27, doi:10.3390/microorganisms9030637.

150. Kanelli, M.; Mandic, M.; Kalakona, M.; Vasilakos, S.; Kekos, D.; Nikodinovic-Runic, J.; Topakas, E. Microbial production of violacein and process optimization for dyeing polyamide fabrics with acquired antimicrobial properties. Front. Microbiol. 2018, 9, 1495, doi:10.3389/fmicb.2018.01495.

151. Poorniammal, R.; Parthiban, M.; Gunasekaran, S.; Murugesan, R.; Thilagavathi, G. Natural dye production from Thermomyces sp fungi for textile application. Indian J. Fibre Text. Res. 2013, 38, 276-279.

152. Venil, C.K.; Velmurugan, P.; Dufossé, L.; Devi, P.R.; Ravi, A.V. Fungal pigments: Potential coloring compounds for wide ranging applications in textile dyeing. J. Fungi 2020, 6, 68, doi:10.3390/jof6020068.

153. Gallego, R.; Alves, M.J.; Tardif, C.; Ibáñez, E.; Parreira, C.; Herrero, M.; Guerra, T. Simultaneous extraction and purification of fucoxanthin from Tisochrysis lutea microalgae using compressed fluids. 2020, 1-11, doi:10.1002/jssc.202000021.

154. Wang, N.N.; Li, C.M.; Li, Y.X.; Du, Z.J. Aquimarina celericrescens sp. Nov., isolated from seawater. Int. J. Syst. Evol. Microbiol. 2018, 68, 1683-1688, doi:10.1099/ijsem.0.002733.

155. Wu, Y.H.; Zhou, P.; Jian, S.L.; Liu, Z.S.; Wang, C.S.; Oren, A.; Xu, X.W. Pontibacter amylolyticus sp. Nov., isolated from a deep-sea sediment hydrothermal vent field. Int. J. Syst. Evol. Microbiol. 2016, 66, 1760-1767, doi:10.1099/ijsem.0.000944.

156. Xamxidin, M.; Wu, Y.H.; Jian, S.L.; Zhou, Y.D.; Wang, C.S.; Tohty, D.; Xu, X.W. Aquaticitalea lipolytica gen. Nov., sp. nov., isolated from antarctic seawater. Int. J. Syst. Evol. Microbiol. 2016, 66, 2657-2663, doi:10.1099/ijsem.0.001101.

157. Neelam, D.K.; Agrawal, A.; Tomer, A.K.; Bandyopadhayaya, S.; Sharma, A.; Jagannadham, M. V.; Mandal, C.C.; Dadheech, P.K. A piscibacillus sp. Isolated from a soda lake exhibits anticancer activity against breast cancer mda-mb-231 cells. Microorganisms 2019, 7, 34, doi:10.3390/microorganisms7020034.

158. Zhou, L.Y.; Yu, Z.L.; Xu, W.; Mu, D.S.; Du, Z.J. Maribellus luteus gen. Nov., sp. nov., a marine bacterium in the family prolixibacteraceae isolated from coastal seawater. Int. J. Syst. Evol. Microbiol. 2019, 69, 2388-2394, doi:10.1099/ijsem.0.003495.

159. Geng, Y.; Zhang, Y.; Qin, K.; Liu, J.; Tian, J.; Huang, Y.; Wei, Z.; Zhang, F.; Peng, F. Sphingomonas paeninsulae sp. Nov., isolated from soil sampled at fildes peninsula, Antarctica. Int. J. Syst. Evol. Microbiol. 2019, 69, 3702-3709, doi:10.1099/ijsem.0.003504.

160. Liu, Y.H.; Fang, B.Z.; Dong, Z.Y.; Li, L.; Mohamad, O.A.A.; Zhang, Y.G.; Egamberdieva, D.; Xiao, M.; Li, W.J. Croceibacterium gen. Nov., with description of croceibacterium ferulae sp. nov., an endophytic bacterium isolated from ferula sinkiangensis k. m. shen and reclassification of porphyrobacter mercurialis as croceibacterium mercuriale comb. nov. Int. J. Syst. Evol. Microbiol. 2019, 69, 2547-2554, doi:10.1099/ijsem.0.003540.

161. Cai, H.; Cui, H.; Zeng, Y.; An, M.; Jiang, H. Sandarakinorhabdus cyanobacteriorum sp. Nov., a novel bacterium isolated from cyanobacterial aggregates in a eutrophic lake. Int. J. Syst. Evol. Microbiol. 2018, 68, 730-735, doi:10.1099/ijsem.0.002571.

162. Chun, B.H.; Lee, Y.; Jin, H.M.; Jeon, C.O. Cloacibacterium caeni sp. nov., isolated from activated sludge. Int. J. Syst. Evol. Microbiol. 2017, 67, 1688-1692, doi:10.1099/ijsem.0.001841.

163. Jia, L.; Feng, X.; Zheng, Z.; Han, L.; Hou, X.; Lu, Z.; Lv, J. Polymorphobacter fuscus sp. nov., isolated from permafrost soil, and emended description of the genus Polymorphobacter. Int. J. Syst. Evol. Microbiol. 2015, 65, 3920-3925, doi:10.1099/ijsem.0.000514. 